\title{
Cotton pan-genome retrieves the lost sequences and genes during domestication and selection
}

Jianying Li ${ }^{1} \mathbb{D}$, Daojun Yuan${ }^{2}$, Pengcheng Wang ${ }^{1}$, Qiongqiong Wang ${ }^{1}$, Mengling Sun ${ }^{1}$, Zhenping Liu', Huan Si ${ }^{1}$, Zhongping Xu', Yizan Ma ${ }^{1}$, Boyang Zhang ${ }^{1}$, Liuling Pei ${ }^{1}$, Lili Tu' ${ }^{1}$ Longfu Zhu' ${ }^{1}$ Ling-Ling Chen ${ }^{3}$, Keith Lindsey ${ }^{4}$, Xianlong Zhang ${ }^{1}$, Shuangxia Jin ${ }^{1 *}$ and Maojun Wang ${ }^{1 *}$ (D)

* Correspondence: jsx@mail.hzau. edu.cn; mjwang@mail.hzau.edu.cn ${ }^{1}$ National Key Laboratory of Crop Genetic Improvement, Huazhong Agricultural University, Wuhan, China

Full list of author information is available at the end of the article

\begin{abstract}
Background: Millennia of directional human selection has reshaped the genomic architecture of cultivated cotton relative to wild counterparts, but we have limited understanding of the selective retention and fractionation of genomic components.

Results: We construct a comprehensive genomic variome based on 1961 cottons and identify $456 \mathrm{Mb}$ and $357 \mathrm{Mb}$ of sequence with domestication and improvement selection signals and 162 loci, 84 of which are novel, including 47 loci associated with 16 agronomic traits. Using pan-genome analyses, we identify 32,569 and 8851 non-reference genes lost from Gossypium hirsutum and Gossypium barbadense reference genomes respectively, of which $38.2 \%(39,278)$ and $14.2 \%(11,359)$ of genes exhibit presence/absence variation (PAV). We document the landscape of PAV selection accompanied by asymmetric gene gain and loss and identify 124 PAVs linked to favorable fiber quality and yield loci.
\end{abstract}

Conclusions: This variation repertoire points to genomic divergence during cotton domestication and improvement, which informs the characterization of favorable gene alleles for improved breeding practice using a pan-genome-based approach.

Keywords: Cotton, Domestication, Improvement, Pan-genome, Copy number variation (CNV), Presence/absence variation (PAV), Gene loss

\section{Background}

Cotton is cultivated worldwide for its fiber and seed oil. Allotetraploid cultivated cotton (Gossypium hirsutum and Gossypium barbadense) originated from interspecies hybridization between putative diploid A genome (Gossypium herbaceum or Gossypium arboretum) and D genome (Gossypium raimondii) ancestors approximately 11.5 million years ago (MYA) [1, 2]. G. hirsutum was initially domesticated from wild cotton in the Yucatan peninsula and subsequently developed seven semi-wild forms, which were subject to directional domestication selection to form the American cultivated cotton with high yield and spinnable fine white fibers [3-6]. DNA-based

(c) The Author(s). 2021 Open Access This article is licensed under a Creative Commons Attribution 4.0 International License, which permits use, sharing, adaptation, distribution and reproduction in any medium or format, as long as you give appropriate credit to the original author(s) and the source, provide a link to the Creative Commons licence, and indicate if changes were made. The images or other third party material in this article are included in the article's Creative Commons licence, unless indicated otherwise in a credit line to the material. If material is not included in the article's Creative Commons licence and your intended use is not permitted by statutory regulation or exceeds the permitted use, you will need to obtain permission directly from the copyright holder. To view a copy of this licence, visit http://creativecommons.org/licenses/by/4.0/. The Creative Commons Public Domain Dedication waiver (http://creativecommons.org/publicdomain/zero/1.0/) applies to the data made available in this article, unless otherwise stated in a credit line to the data. 
molecular markers have revealed admixture population structure and high genetic diversity of G. hirsutum wild, landrace, and American cultivated cotton [7-10]. Founder cultivars of G. hirsutum in America have been introduced widely to other countries and improved modern cultivars in China show wide phenotypic variation and adaptation [3-6]. G. barbadense is native to the coastal areas of Peru and is cultivated in a limited number of areas with superior fibers [11]. The history of allotetraploid cotton domestication and selection has been revealed at small-scale variation level in specific population, but the understanding of entire genomic variome remains fragmentary.

Genome assemblies of G. hirsutum and G. barbadense cultivars have identified extensive variation between the species $[12,13]$. Hundreds of diverse cotton accessions have been sequenced, providing an opportunity to construct a multi-dimensional variation genome (variome) to reveal genome divergence during domestication and identify loci underlying improvement traits [4-6, 14-16]. However, these genomic variations were identified by sequence reads mapped to a reference genome, giving an incomplete picture, especially for the lack of presence/absence variation (PAV) and copy number variation $(\mathrm{CNV})$. To comprehensively capture the genetic variation missed by using one reference, the construction of a "pan-genome," a collection of all the DNA sequences from all individuals in a species, has a great value [17]. Pan-genomic studies can identify PAVs between wild and cultivated accessions for a better understanding of crop domestication [18-31]. In tomato, $351 \mathrm{Mb}$ non-reference sequences with 4873 novel genes, including $74 \%$ for core genes (present in all accessions) and $26 \%$ for dispensable genes (present in at least one accession), were assembled using 725 representative wild and improved accessions [26]. In soybean, based on long reads of 27 wild and cultivated soybean accessions, pan-genome assembly revealed that $36 \%$ and $64 \%$ of genes were core and dispensable respectively, some of which were associated with domestication traits [30]. These studies suggest that PAVs are widespread and play an important role in genetic determination of phenotypic variation [32], to reveal favorable genotypes for crop improvement.

Here we analyze genomic variation among 1961 cottons, revealing extensive genomic diversity, including 63 million single-nucleotide polymorphisms (SNPs), 4.9 million small insertion/deletions (InDels), and over 290,000 structural variations (SVs). We constructed pan-genomes of G. hirsutum and G. barbadense, which include $1041 \mathrm{Mb}$ (32,569 genes) and $309 \mathrm{Mb}$ (8851 genes) non-reference sequences, respectively. The domestication and improvement process has led to asymmetric gene gain and loss, which shaped the genomic architecture of cultivated cotton. The pan-genome data inform us to understand how domestication and improvement has driven genomic picture underlying the desirable agronomic traits for further cotton breeding.

\section{Results}

\section{Genetic diversity and population properties}

We collected DNA re-sequencing data for 1961 cottons for a genomic variation analysis with an average depth of $\sim 14.8 \times$ for each [3-6, 16, 33, 34]. After discarding duplicated accessions, a total of 1913 cotton accessions were used for SNP and InDel analysis, which included 256 G. hirsutum landraces (Ghlandraces), 438 improved G. hirsutum cultivars from the USA and other countries (GhImpUSO), 929 improved G. 
hirsutum cultivars from China (GhImpCHN), 261 G. barbadense accessions, and 29 other Gossypium species that were used as outgroup (Additional file 1: Table S1). We aligned these data against the reference genome of G. hirsutum acc. "TM-1" [12] and identified 63,084,975 SNPs and 12,354,432 small insertions or deletions (InDels length $\leq 20 \mathrm{bp}$ ), in which the core variation dataset includes 19,246,497 SNPs and 4,815, 125 InDels with a minor allele frequency (MAF) $\geq 0.01$ and more than five accessions having homozygous variations (Table 1; Additional file 1: Tables S2-S6; Additional file 3). Based on core SNP data, we investigated the population structure of G. hirsutum and G. barbadense. Neighbor-joining tree analysis showed the 1913 accessions classify into 12 clades. G. hirsutum accessions form 8 clades, G. barbadense accessions form 3 clades, and other species form 1 clade (Fig. 1a; Additional file 2: Figure S1). Population analysis showed that G. barbadense accessions were separated from the G. hirsutum landraces, GhImpUSO and GhImpCHN (Fig. 1b, c; Additional file 2: Figure S2). G. hirsutum nucleotide diversity $(\pi)$ is estimated at $1.07 \times 10^{-3}$ in landraces, $3.74 \times 10^{-4}$ in GhImpUSO, $3.34 \times 10^{-4}$ in GhImpCHN, and $1.01 \times 10^{-3}$ in G.

Table 1 Genome-wide genomic variations in a large cotton population

\begin{tabular}{|c|c|c|c|c|c|c|c|}
\hline Variation type & $\begin{array}{l}\text { Total } \\
\text { (1913) }\end{array}$ & $\begin{array}{l}\text { Gh cultivar } \\
\text { (1623) }\end{array}$ & $\begin{array}{l}\text { Ghlandrace } \\
\text { (256) }\end{array}$ & $\begin{array}{l}\text { GhlmpUSO } \\
\text { (438) }\end{array}$ & $\begin{array}{l}\text { GhlmpCHN } \\
\text { (929) }\end{array}$ & $\begin{array}{l}\text { Gb cultivar } \\
(261)\end{array}$ & $\begin{array}{l}A D_{3}-A D_{7} \\
(26)\end{array}$ \\
\hline$\overline{\text { Bi-allele SNPa }}$ & $\begin{array}{l}19,246 \\
497\end{array}$ & $9,546,748$ & $9,265,438$ & 4766,399 & $3,761,448$ & $19,473,033$ & $\begin{array}{l}32,878 \\
758\end{array}$ \\
\hline Splicing & 2172 & 1213 & 1149 & 652 & 554 & 2041 & 11,366 \\
\hline Exonic & 315,404 & 179,665 & 172,718 & 103,126 & 89,208 & 316,146 & 776,644 \\
\hline Intronic & 607,301 & 335,212 & 322,141 & 189,798 & 152,656 & 575,524 & $1,010,509$ \\
\hline UTR & 220,664 & 120,198 & 116,269 & 65,226 & 52,342 & 197,420 & 390,008 \\
\hline Upstream & 869,678 & 448,709 & 432,640 & 238,937 & 169,788 & 789,898 & 984,811 \\
\hline Downstream & 797,469 & 413,937 & 399,140 & 222,266 & 161,602 & 729,445 & 959,584 \\
\hline Nonsynonymous & 195,883 & 111,686 & 107,143 & 63,008 & 52,853 & 177,474 & 420,190 \\
\hline InDel ( $\leq 20 \mathrm{bp})^{\mathrm{a}}$ & $\begin{array}{l}4,815 \\
125\end{array}$ & $3,971,277$ & $3,744,299$ & $1,672,195$ & $1,726,445$ & $3,366,481$ & $7,625,077$ \\
\hline Splicing & 1202 & 1128 & 941 & 570 & 735 & 1104 & 2465 \\
\hline Exonic & 31,661 & 27,238 & 28,815 & 12,807 & 14,826 & 26,455 & 65,677 \\
\hline Intronic & 262,657 & 231,561 & 215,663 & 95,674 & 94,830 & 183,387 & 539,379 \\
\hline UTR & 113,824 & 100,811 & 96,003 & 36,418 & 37,175 & 76,684 & 261,351 \\
\hline Upstream & 578,086 & 497,660 & 413,192 & 201,122 & 226,848 & 400,965 & 927,134 \\
\hline Downstream & 429,514 & 369,517 & 311,164 & 148,050 & 166,059 & 309,829 & 717,980 \\
\hline Frameshift & 23,330 & 20,367 & 22,040 & 9798 & 11,029 & 19,603 & 42,328 \\
\hline SV (> 50 bp) & 214,310 & 104,523 & 97,933 & 64,064 & 61,616 & 132,499 & 281,476 \\
\hline Deletion $^{\mathrm{b}}$ & 32,099 & 22,340 & 9933 & 7029 & 23,559 & 13,982 & 15,484 \\
\hline Duplication ${ }^{\mathrm{b}}$ & 7576 & 5146 & 4766 & 1721 & NA & 3252 & 3718 \\
\hline Inversion $^{b}$ & 1112 & 724 & 615 & 310 & NA & 877 & 613 \\
\hline Translocation $^{\text {b }}$ & 357 & 240 & 188 & 167 & NA & 504 & 412 \\
\hline $\mathrm{CNV}^{\mathrm{C}}$ & 173,166 & 76,073 & 82,431 & 54,837 & 38,057 & 99,274 & 261,249 \\
\hline
\end{tabular}

${ }^{\text {a The }} 261$ G. barbadense accessions were aligned to the "TM-1" reference genome. The G. barbadense population SNP and InDel calling results against the "3-79" reference genome are shown in Additional file 1: Table S5. 'benotyping structural variations (SVs) in 742 cottons. The G. hirsutum TM-1 reference genome was used for detecting variations. The number of genotypes in each group is in parentheses. "NA" represents the missing combined SVs. DUP, INV, and TRA were not included for the GhImpCHN population. ${ }^{\mathrm{C}} \mathrm{CNV}$ s were identified in 742 cottons. Only variation in each chromosome was counted and further analyzed 


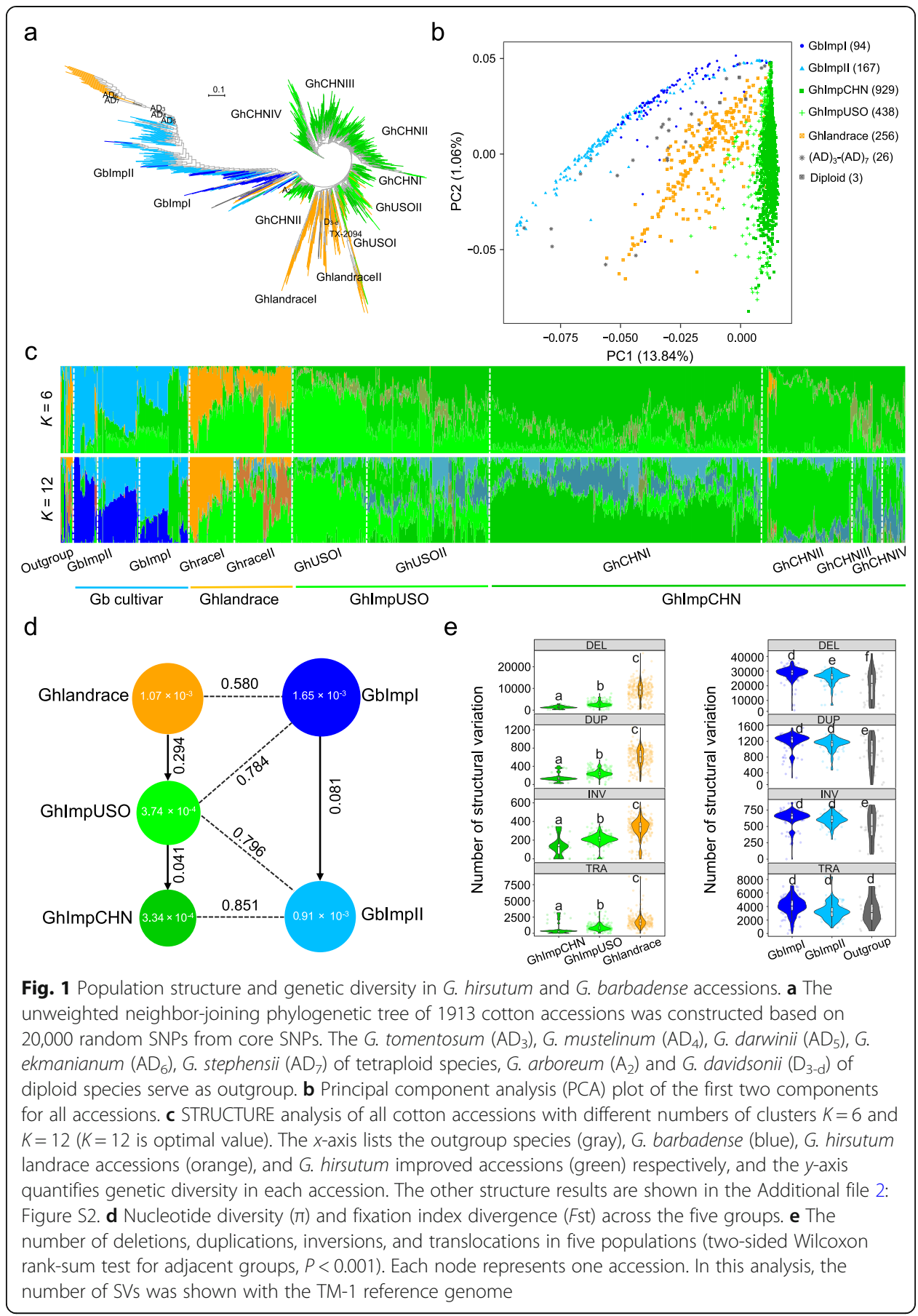

barbadense (Additional file 2: Figure S3), similar to the recent studies in cotton [3-6, 34] (Fig. 1d).

We used 742 cotton accessions with a high sequencing depth (>10x) against the G. hirsutum "TM-1" reference genome (Additional file 1: Table S1; Additional file 3) and identified 32,099 deletions, 7576 duplications, 1112 inversions, and 357 translocations (Additional file 1: Table S7). There are more SVs in Ghlandrace than that GhImpUSO and GhImpCHN groups (Fig. 1e). In addition, 173,166 (MAF $\geq 0.01$ ) copy number variations (CNVs) were identified in the 742 accessions, including 82,431 in the landraces, 
59,309 in the GhImpUSO, and 38,057 in the GhImpCHN group (Additional file 1: Table S8). Population genetic properties of CNVs in 742 accessions showed that G. hirsutum landraces were clearly separated from the improved accessions, similar to SNPbased result, but were clustered together with the GhImpUSO and GhImpCHN accessions (Additional file 2: Figure S4). These results suggested that high-confidence CNVs have strong divergence between G. hirsutum landrace and improved population and can be used to discover complex quantitative trait loci (QTLs). This comprehensive variome dataset provides a genomic resource for cotton population genetics, domestication analysis, and agronomic allele identification (Additional file 2: Figure S5).

\section{Evidence for genomic divergence during domestication and improvement}

Domestication-related traits arise from selected genetic variation in wild species, affecting seed size, flowering time, yield, quality, and crop adaptation [35-37]. To identify potential selection signals during cotton domestication, we scanned genetic variations with allele frequency differentiation in nucleotide diversity by comparing each cultivated group with its corresponding wild group. We identified 76 domestication sweep regions (DSRs) using $\pi_{\text {Landrace }} / \pi_{\text {Improved }}($ ratio $\geq 15$ ) and a likelihood method (XP-CLR, Top 5\%) (Additional file 2: Figure S6a), occupying $66.8 \mathrm{Mb}$ in the A subgenome and $51.4 \mathrm{Mb}$ in the $\mathrm{D}$ subgenome associated with 837 and 1272 genes, including 274 homologous gene pairs (Fig. 2a). Compared with previous studies with small numbers of accessions [3-5], this domestication selection analysis identified 31 novel DSRs occupying 43.6 Mb (Additional file 1: Table S9). Some fiber-related and known domesticated genes were differentially expressed between wild/landraces and improved cultivars (Additional file 2: Figure S6b, c). The domestication selected genes were involved in stress response, cell wall regulation, jasmonic acid, ethylene, and circadian rhythm process (Additional file 2: Figure S7). Further manipulation of these genes in plant hormone pathway and stress response pathway may help illustrate their putative regulatory role in fiber quality improvement and environmental adaptation during cotton domestication $[3,38,39]$. We also identified $120 \mathrm{Mb}\left(\pi_{\mathrm{GhImpUSO}} / \pi_{\mathrm{GhImpCHN}} \geq 2\right)$ with improvement signals, including 1006 selected genes in the A subgenome and 2369 in the D subgenome with 353 homologous gene pairs (Fig. 2a; Additional file 2: Figure S6d), and $79.5 \%(95.4 \mathrm{Mb})$ of the improvement selection regions were not identified previously [5] (Additional file 1: Table S10). Of note is the observation that $19 \mathrm{Mb}$ of sequence was screened with both domestication and improvement selection signals, in which the D subgenome (441 genes) has more genes than the A subgenome (50 genes) (Additional file 1: Table S11). These data suggest that D subgenome has stronger SNP-based selection signals in both domestication and improvement processes.

Domestication is a driver for $\mathrm{CNV}$ allele frequency difference between wild/landrace and domesticated groups [37]. In total, 286 non-redundant CNV-based regions were identified with selection signals during cotton domestication, comprising $297 \mathrm{Mb}$ in the A subgenome (Fig. 2b) and $105 \mathrm{Mb}$ in the D subgenome (Fig. 2f). About 55\% (65 Mb of $118 \mathrm{Mb}$ ) of SNP-based domestication signals overlapped CNV-based domestication sweeps (Additional file 1: Table S12). In total, 217 CNV regions were identified with improvement selection signals, comprising $156 \mathrm{Mb}$ in the A subgenome and $133 \mathrm{Mb}$ in the D subgenome. About $44 \%$ (52 Mb of $120 \mathrm{Mb}$ ) of SNP-based improvement signals 


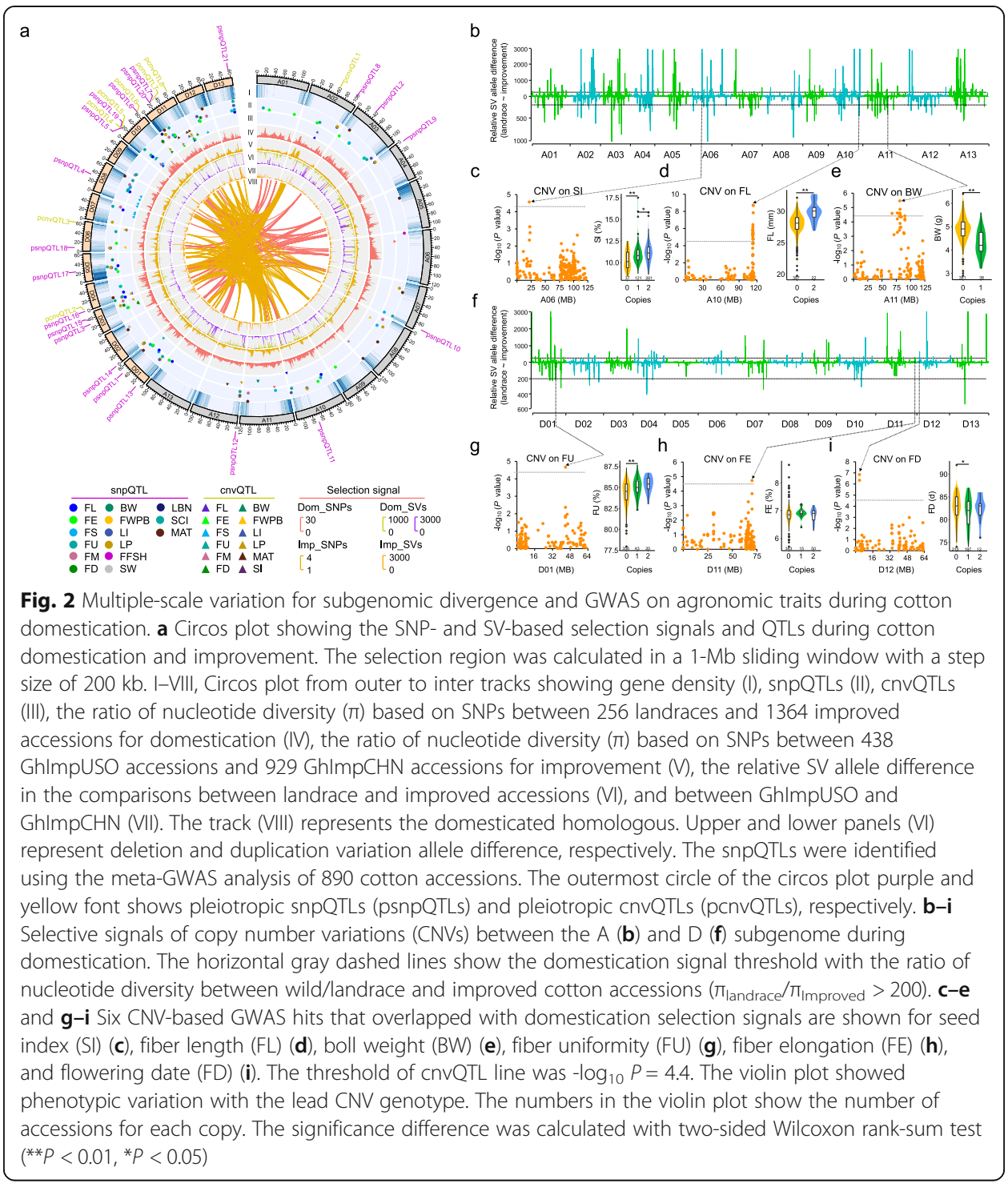

overlapped the CNV-based improvement signals (Additional file 1: Table S13). In total, we identified $329 \mathrm{Mb}$ (covering 6339 genes) of sequences in the A subgenome and 127 $\mathrm{Mb}$ (4955 genes) in the D subgenome with both SNP- and CNV-based domestication signals. A total of $173 \mathrm{Mb}$ (5526 genes) and $184 \mathrm{Mb}$ (8405 genes) of sequences have improvement signals in the A and D subgenomes. The identification of selection signals during domestication and improvement can facilitate to further identify genetic loci of important agronomic traits.

To identify QTLs for selection signals associated with agronomic traits, we conducted a genome-wide association study (GWAS) meta-analysis of 890 G. hirsutum accessions from three independent experimental cases with multiple environments (Additional file 3) $[3,5,6]$. Using the genotypic data of 2,291,437 high-quality SNPs with MAF $\geq 0.05$ in 890 accessions, we identified 2952 significant SNPs $\left(0.05 / 2,291,437 ; P<2.18 \times 10^{-8}\right)$ associated with fiber quality-related traits. After strict filtering, 91 major fiber-related QTLs were located, including 11 for fiber length (FL), 17 for fiber elongation (FE), 15 for fiber strength (FS), 19 for fiber length uniformity (FU), 10 for fiber micronaire 
(FM), 7 for fiber maturity (MAT), and 12 for spinning consistency index (SCI) (Additional file 1: Table S14 and Additional file 2: Figure S8). We also identified 31 yieldrelated and 3 flowering date (FD)-related QTLs. In total, 125 major QTLs with 4751 candidate genes for 15 agronomic traits were identified, in which 78 were consistent with previous studies $[3,5,6,15,40,41]$ and the other 47 were newly detected in meta-analysis (Additional file 1: Table S14). In the 125 QTLs, 14 have selection signals during domestication and improvement (Additional file 1: Table S15). In addition, twenty-one QTL loci showed pleiotropic effects on fiber quality, yield, and flowering date (Fig. 2a; Additional file 1: Table S16). For example, lint percentage (LP), fiber weight per boll (FWPB), and lint index (LI) are components of yield trait, with major QTLs co-localized on chromosome D02 (Additional file 2: Figure S9a). The LP, FD, and whole growth period (WGP) for flowering time traits have co-located QTLs on chromosome D03 (Additional file 2: Figure S9b).

We focused on novel QTLs related to fiber elongation that were identified in metaGWAS. A novel QTL (mqFE253) was located on the D05 chromosome (at 11.3-12.5 $\mathrm{Mb}$ of genomic region). The 64 candidate genes were predicted by integrating haplotype analysis, gene expression, and functional annotation (Additional file 2: Figure S10). One candidate gene (Ghir_D05G013680, GhIDD7), encoding an indeterminate-domain 7 transcription factor, was differentially expressed in four fiber developmental stages (Additional file 2: Figure S10f). Accessions representing two main haplotypes of the 5' UTR region showed a significant difference in fiber elongation and fiber length (Additional file 2: Figure S11a-b). After knock-out of GhIDD7, the mature fiber was significantly shorter than that in wild type plants $(25.8 \pm 0.3$ vs. $27.1 \pm 0.1)$ (Additional file 2: Figure S11c, d, e). These results indicated that GhIDD7 was a previously uncharacterized gene contributing to fiber quality-related trait.

GWAS analysis of 26,831 high-confidence CNVs (MAF $\geq 0.05$ ) in 419 G. hirsutum accessions revealed 370 significant CNVs for 50 QTLs (cnvQTLs) (Additional file 1: Table S17), of which 5 showed pleiotropic effects on both fiber quality and lint yield (Fig. 2a). Thirteen cnvQTLs overlapped with SNP-based QTLs (snpQTLs), and the other 37 cnvQTLs are only identified by CNVs. Of these cnvQTLs, 15 overlapped with domestication sweeps and 10 overlapped with improvement selection signals (Additional file 1: Table S18). The phenotypic data exhibit a significant difference in cotton accessions with different copy numbers of lead CNV (Fig. 2c-e, g-i; Additional file 2: Figure S12). For example, a seed index (SI) association with domestication signal was identified on the A06 chromosome (Fig. 2c). A fiber length (FL) association with domestication signal was located on the A10 chromosome, and FL with 2 duplication copies was significantly longer than that with 0 copy (reference) allele $(P<0.01)$ (Fig. 2d). The lead CNV-involved LD region has 78 candidate coding genes, in which some are involved in cotton fiber development, such as UDP-glucose pyrophosphorylase 3 (Ghir_A10G024310, UGP3) and AP2/B3-like transcriptional factor (Ghir A10G023950). Another example shows a fiber maturity (MAT) association with improvement selection signal was located on the A12 chromosome (Additional file 2: Figure S13a, b, c). This association has one candidate gene encoding xyloglucan endotransglucosylase/hydrolase 5 (Ghir_A12G008500, XTH5). In the D subgenome, three cnvQTLs with strong selection signals were found to be associated with FD, FWPB, and FS on the D03, D06, and D07 chromosomes (Additional file 2: Figure S13d, 
e, f, g). These results provide a number of cnvQTL candidates that may be applied to cultivate desirable traits in future breeding.

\section{Pan-genomes of G. hirsutum and G. barbadense species}

We used a reference-guided assembly approach [21] to construct pan-genomes of $G$. hirsutum and G. barbadense. The sequencing data of 1581 G. hirsutum (251 landraces, 424 GhImpUSO and 906 GhImpCHN) and 226 G. barbadense improved accessions were aligned to the "TM-1" and " $3-79$ " reference genomes, respectively [12]. About 5800 million unmapped reads from G. hirsutum and 1127 million unmapped reads from G. barbadense were subject to de novo assembly (Additional file 2: Figure S14, S15), producing $5,047,083,790 \mathrm{bp}$ and $1,517,253,311 \mathrm{bp}$ of contig sequence respectively, with a minimum length of $500 \mathrm{bp}$ (Additional file 1: Table S19). After removing redundancies, $3704 \mathrm{Mb}$ and $1422 \mathrm{Mb}$ non-reference sequences with a contig N50 of $1530 \mathrm{bp}$ (G. hirsutum) and $1108 \mathrm{bp}$ (G. barbadense) passed all filtering steps for the final nonreference genomes (Additional file 1: Table S20). The final $1041 \mathrm{Mb}$ and $309 \mathrm{Mb}$ nonreference sequences in G. hirsutum and G. barbadense with a contig length of more than $1000 \mathrm{bp}$ were used for predicting protein-coding genes (Additional file 2: Figure S16). We obtained 32,569 G. hirsutum genes (65,679 transcripts) and 8851 G. barbadense genes (12,076 transcripts) (Additional file 1: Tables S21-S22). The final G. hirsutum pan-genome (Ghpan-genome) is $3388 \mathrm{Mb}$ with 102,768 genes (2347 Mb with 70 , 199 genes in the "TM-1" reference genome) and G. barbadense (Gbpan-genome) is $2575 \mathrm{Mb}$ with 80,148 genes (2266 Mb with 71,297 genes in the "3-79" reference genome) (Additional file 2: Figure S17).

The coverage of the Ghpan-genome was investigated using PacBio reads of 10 representative accessions, including G. hirsutum yucatanense, G. hirsutum richmondi, G. hirsutum morrilli from the wild/landraces, the Acala, Paymaster 54, Stoneville 2B from the GhImpUSO group, and Simian 3, CRI 7, Xinluzao 42, and Xuzhou 142 from the GhImpCHN group (Additional file 1: S23-S25; Additional file 2: Figure S18). After de novo assembly (Additional file 3), more than 93\% of assembled contigs were mapped to the TM-1 reference genome. Approximately $18.9 \mathrm{Mb}$ of unmapped contigs (a total of $641 \mathrm{Mb}$ contigs from 10 accessions that were not mapped on the TM- 1 reference genome) were aligned to the non-reference sequences of $1581 \mathrm{G}$. hirsutum accessions (the average non-reference sequence length is $\sim 655 \mathrm{~kb} ; 1041 \mathrm{Mb} / 1581 \mathrm{Mb})$. The PacBiobased assemblies provide evidence for non-reference genome sequences in G. hirsutum, indicating that our pipeline of pan-genome construction can retrieve PAVs in a large germplasm population. Some high-frequency PAVs were also verified by PCR in 23 representative accessions (Additional file 2: Figure S19).

For the G. hirsutum population, we mapped re-sequencing reads against 102,768 pan genes, which resulted in 17,100 genes (16.64\%, singleton) in 561 accessions (sequencing depth $<5$ ) and 85,667 genes in 1020 accessions (depth $>5$ ). The 1020 G. hirsutum accessions include 63,489 core genes shared by all G. hirsutum accessions, 5941 (5.78\%) softcore genes in 990-1019 accessions (97-100\%), 3803 (3.7\%) shell genes in 11-989 accessions (1-97\%), and 12,434 (12.1\%) clouds in less than 10 accessions (0-1\%) (Fig. 3a, b). For the G. barbadense pan-genome, the 1536 singleton genes only occurred in 49 low-depth accessions. We used 78,612 pan genes that occurred in 177 accessions 
for further PAV analysis. The 177 G. barbadense accessions include 68,789 (85.8\%) core genes, 1796 (2.24\%) softcore genes in 172-176 accessions (97-100\%), 5867 (7.32\%) shell genes in 4-171 accessions (2-97\%), and 2160 (2.75\%) clouds in less than 3 accessions (0-2\%) (Fig. 3c, d). Modeling of pan-genome size with iteratively random sampling suggests that the Ghpan-genome has an average of 81,688 pan genes and an average of 65,595 core genes in 398 accessions (Fig. 3e). The Gbpan-genome has an average of 78,607 pan genes and 69,563 core genes in 59 accessions for modeling saturation (Fig. 3f). Therefore, the size of core-genome decreased and pan-genome increased with the increase of population size. GO analysis showed that core genes were involved in cellular metabolic process and development, whereas the variable genes were involved in "defense response," "response to stress," and "signaling transduction in environment fitness" (Additional file 2: Figure S20).

We next investigated the genomic characteristics of core and variable genes between A and D subgenome. Core genes have higher expression levels than variable genes in both G. hirsutum and G. barbadense (Additional file 2: Figure S21). Interestingly, A subgenomic variable genes have higher expression levels than $D$ subgenomic genes (Fig. 4a). Variable genes have a higher adjacent $(2 \mathrm{~kb})$ TE insertion probability than core genes, especially for the Gypsy class (Additional file 2: Figure S22). The variable genes in the D subgenome have a higher ratio than those in the A subgenome (Fig. 4b). Evolutionary selection analysis showed that more variable genes have undergone positive selection than core genes in both G. hirsutum and G. barbadense, especially in the D subgenome (Fig. 4c). Furthermore, variable genes have a larger nucleotide diversity than core genes, and more variable genes in the $\mathrm{D}$ subgenome have a higher diversity $(P<0.001)$ (Fig. 4d; Additional file 2: Figure S23). These data indicated that D subgenomic variable genes had a faster evolutionary rate than A subgenomic genes.

\section{PAV selection during domestication and improvement}

To establish landscape of selective PAVs between landrace and improved cotton, we compared PAV frequency between the landrace, GhImpUSO, and GhImpCHN groups. The landrace group has more variable genes than improved cultivars, suggesting a general trend of gene loss during cotton domestication (Fig. 5a). PCA and phylogenetic analysis of PAVs suggest that the landrace group was separated from the improved cultivar group (Fig. 5b, c). The landraces originating from native America had a population mixture with American cultivated cotton in genetic composition, consistent with the clustering analysis of high-confidence SNPs (Additional file 2: Figure S24). To control the false-positive rate, eight landraces and thirty-four GhImpUSO accessions in a mixed population structure with uncertain origin were excluded from further analysis.

To identify PAV-related genes with selection signals during domestication and improvement, we performed two comparisons between 182 landraces and 206 GhImpUSO accessions using the presence frequency of variable genes, for "domestication" (Fig. 5d; Additional file 2: Figure S25), and between 206 GhImpUSO and 592 GhImpCHN accessions for "improvement" (Fig. 5e). The genes with a significant change of presence frequency ( $F D R<0.001$ and frequency fold change $>2$ for "unfavorable gene" or $<0.5$ for "favorable gene") were regarded as selected genes. Genes with higher presence frequency in landrace than in GhImpUSO, and higher presence 


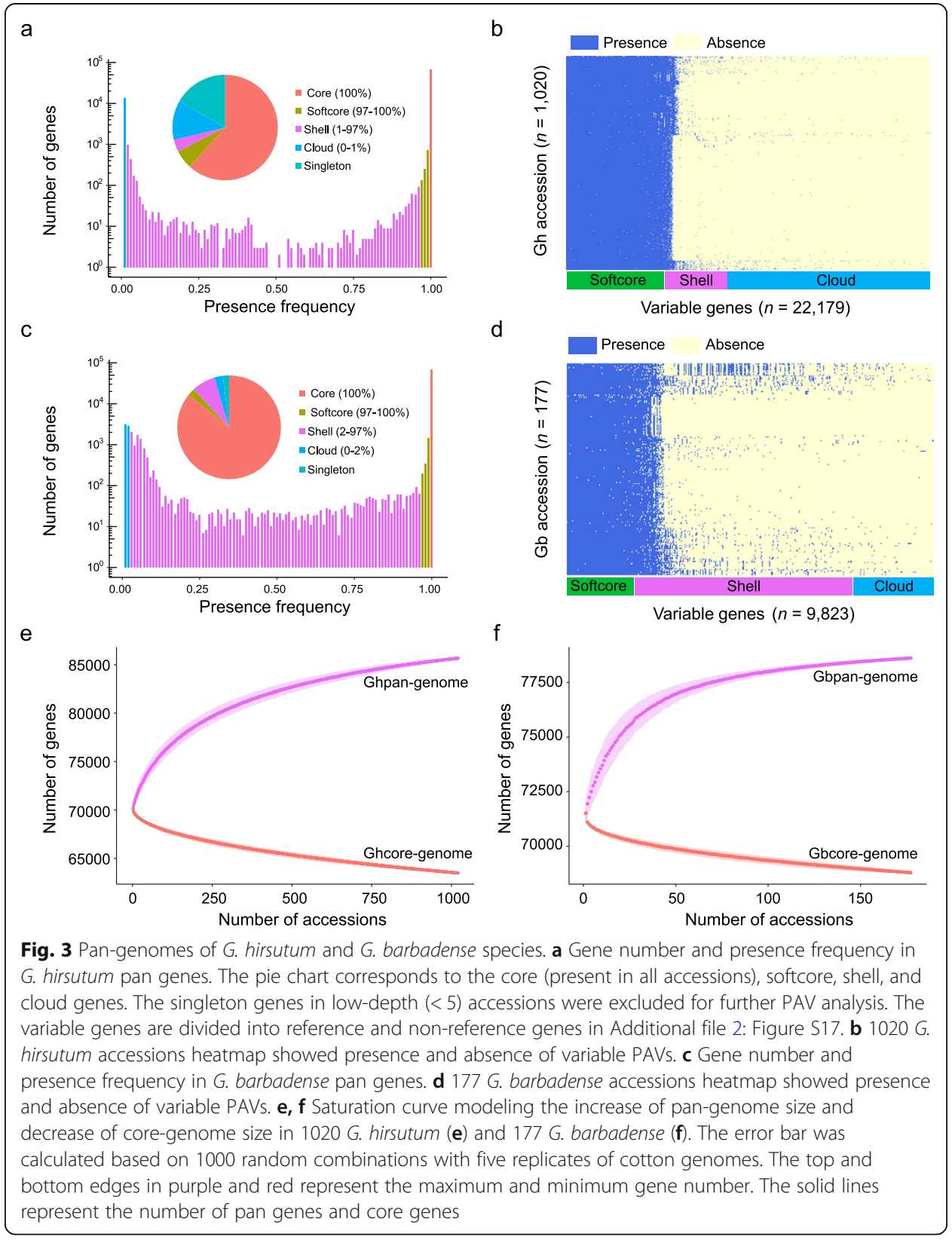

frequency in GhImpUSO than in GhImpCHN were potentially "unfavorable gene," while genes with reverse patterns of presence frequency were "favorable gene." We identified 2785 and 7867 favorable genes with allele gain, and 6753 and 3866 unfavorable genes with allele loss during domestication and improvement, respectively (Additional file 1: Tables S26, S27). GO enrichment analysis showed that favorable genes were enriched in oxidation-reduction-related process, whereas unfavorable genes were enriched in fatty acid biosynthesis and gene regulation. The favorable and unfavorable genes were divided into four comparisons according to the presence frequency in three groups during domestication and improvement (Fig. 5f). The continuous selection of 337 favorable genes with both domestication and improvement signals may be elite candidates for breeding, whereas 308 unfavorable genes exhibiting lower presence 


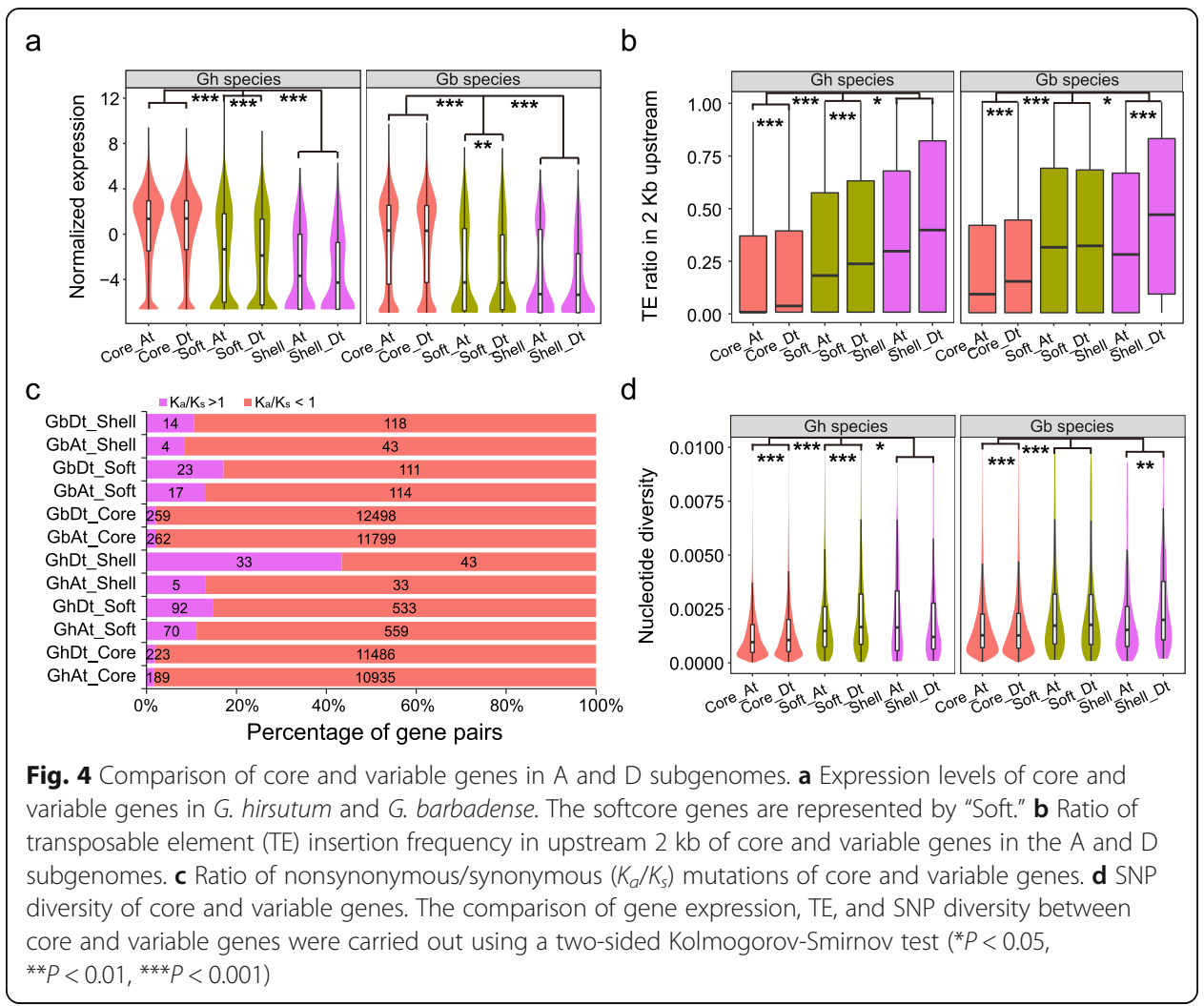

frequencies in the GhImpCHN group represent loss alleles (Fig. 5g; Additional file 1: Table S28). More unfavorable genes than favorable were eliminated during cotton breeding (Fig. 5h). Favorable gain genes participated in transmembrane transport and oxidation-reduction process, whereas favorable loss genes involved in electron transport chain and secondary metabolic process (Fig. 5i, j). Unfavorable gain genes had no significantly enriched process during improvement (Fig. 5j). These analyses showed that many unfavorable gene were lost during domestication and considerable favorable genes were retained during improvement process.

\section{Genes for related traits using pan-genome dataset}

Based on the above data, we propose a summary chart for cotton natural selection, domestication, and improvement (Fig. 6a). We identified nearly $456 \mathrm{Mb}(19.4 \%$ of the assembled reference genome) and $357 \mathrm{Mb}(15.2 \%)$ of sequences with domestication and improvement signals, through the integrated SNP, CNV, and PAV maps (Additional file 1: Table S29). There are 21,169 genes located in domestication regions, some of which have been demonstrated to be involved in the regulation of flowering date, morphology, and fiber development. For the flowering date, a significant GWAS peak on chromosome D03 has two candidate genes encoding a COP1-interactive protein [6] (CIPI, Ghir_D03G008950) and a CONSTANS-like protein [42] (COL2, Ghir_D03G011010), which are required for adaptation change in landrace cotton to cultivated varieties in different geographical areas with different photoperiods. Further investigation of causal SNP alleles shows that the ancestral alleles are mainly distributed in landraces, with 


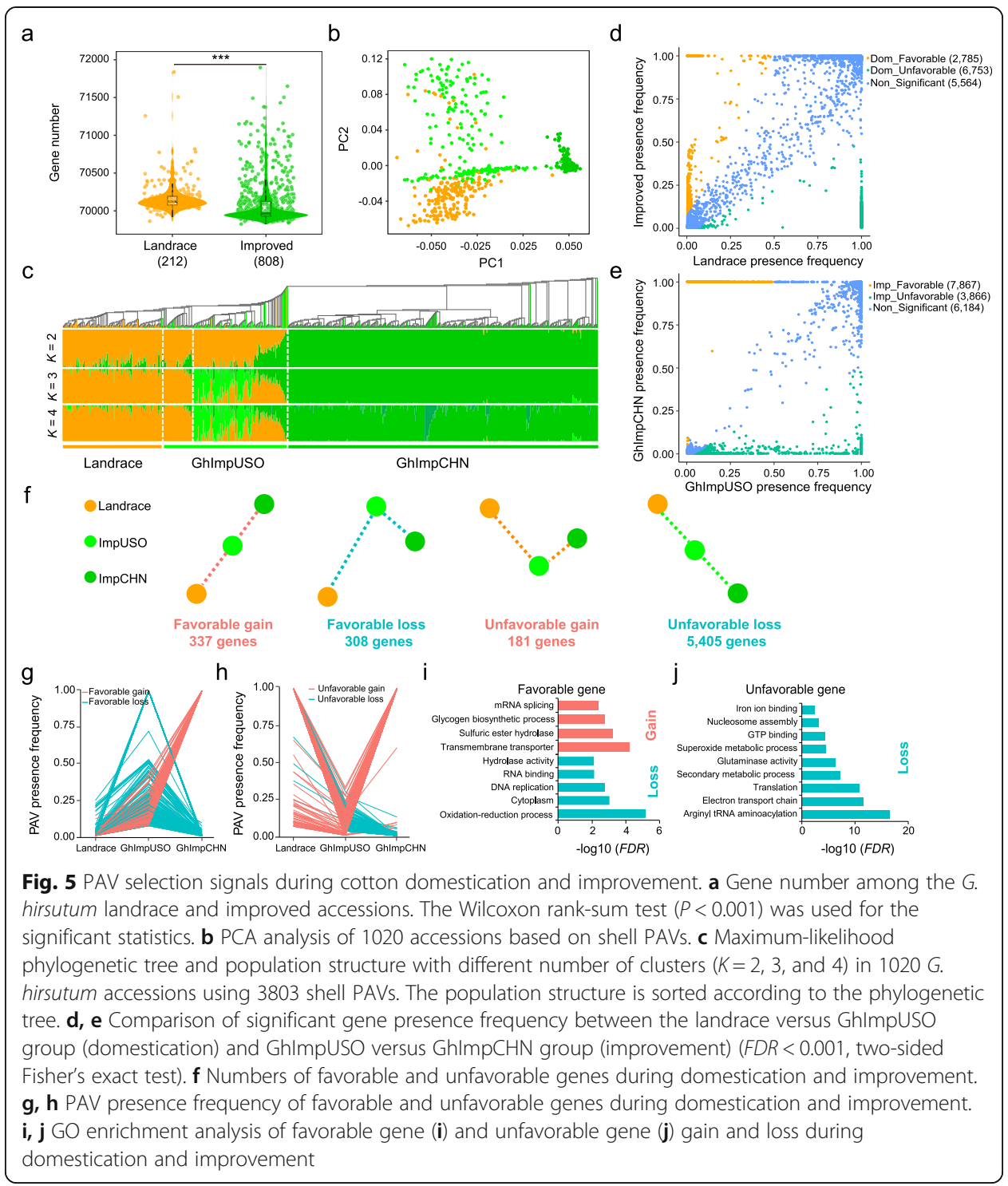

lower allele frequencies in improved cultivars (Fig. 6b). Similarly, we found that landrace and improved groups exhibited allele differentiation in LATE MERISTEM IDENTI TY1 [43] (LMI1, Ghir_D01G021810) that regulates leaf shapes, and in the basic helixloop-helix protein gene GRF (Ghir_A12G025340) that is a candidate gene for cotton glandular QTL [44] (Fig. 6b). Some genes responsible for fiber development that experienced domestication and improvement selection were also detected by the geographical differentiation analysis. KCS2 (Ghir_D10G015750) and CesA6 (Ghir_D03G004880), responsible for fiber elongation [45-48], were subject to domestication and improvement selection (Fig. 6b). The domestication gene PRF3 (Ghir_D13G021640) has a strongly mutated allele in improved cultivars [49].

Pan-genome analysis uncovered favorable and unfavorable gene alleles during domestication and improvement, providing novel candidate genes for functional investigation (Fig. 5). For genes favorable to cotton improvement selection, $S C D$ (short chain dehydrogenase, GhirPan.00056999), ST (sugar transporter, GhirPan.00054328), and RbfA (ribosome-binding factor A, GhirPan.00033905) have the lowest frequency in wild 
population and highest in domesticated cultivars (Fig. 6c; Additional file 2: Figure S26). Some favorable genes exhibiting a decrease of frequency in the improvement process could be eliminated (308 genes), having almost the same allele frequency between wild and cultivated accessions, such as DXS (deoxyxylulose-5-phosphate synthase, Ghir Scaffold1882G000030) and COX3 (cytochrome oxidase subunit 3, Ghir_Scaffold1273G00008). Genes unfavorable during domestication showed increased (182 genes) or decreased (5405 genes) frequency in the GhImpCHN group, such as RLP9 (receptor like protein 9, Ghir_D13G022380) and ZBD (Zinc-binding dehydrogenase, GhirPan.00044196) (Fig. 6c).

To determine the contribution of PAV to agronomic traits, we identified PAVassociated SNPs for 1196 PAVs (MAF $\geq 0.02)$ in 415 accessions (4 accessions were discarded from 419) using 1,904,926 SNPs and obtained 56,486 significant SNPs $(P<$ $\left.2.62 \times 10^{-8}\right)$ associated with 864 (72.2\%) PAVs. Of these PAVs, 124 were overlapped with 89 trait-QTLs (Additional file 1: Table S30; Additional file 2: Figure S27). One representative PAV (Ghir_A08G006710, 543 bp, an uncharacterized gene in G. hirsutum) is located on chromosome A08 (Fig. 6d, Additional file 2: Figure S28). This hotspot region contained two yield-related (LP, FWPB) QTLs and two fiber quality-related (FM, FS) QTLs (Fig. 6e). These accessions with the presence haplotype of this gene showed significantly increased appearance of LP and FWPB traits than those with the absence haplotype, but no difference for FS and FM traits (Fig. 6f). Further presence frequency analysis showed that Ghir_A08G006710 was present in nearly all landrace and GhImpUSO accessions, but was absent in only a few GhImpCHN accessions (Fig. 6g). Interestingly, in the population RNA-Seq data of 15 DPA fiber [15], absence of this gene in 18 accessions was accompanied by significant low expression of an adjacent gene Ghir_A08G006730 (locating at upstream $\sim 61 \mathrm{~kb}$, encoding an AUX/IAA transcriptional regulator family protein) compared with that representing presence of this gene in 233 accessions, supported by the change of IAA content in fibers of representative accessions (Additional file 2: Figure S29, S30). These results implied that this gene represented a recent loss event with a potential regulatory role in other gene expression during cotton improvement. These PAV localization and QTL analyses may improve the efficiency of identifying favorable genes associated with desirable agronomic traits.

\section{Discussion}

Crop domestication and improvement can alter the extent of genomic variation associated with agronomic traits [35-37]. Previous GWAS analyses identified a number of SNP-based genetic loci (snpQTLs) associated with fiber quality, fiber yield, and flowering date in cotton $[3,5,6,14,15]$. Following recently published reference-grade genomes for G. hirsutum with "TM-1" and G. barbadense with "3-79" [12], in this study, we constructed an integrated genomic strategy to construct variome. Using the variome data, we identified 47 novel SNP-based QTLs and 37 CNV-based QTLs, suggesting the power of QTL identification using a larger collection of genomic data. We found that $\sim 19.4 \%(456 \mathrm{Mb})$ and $\sim 15.2 \%(357 \mathrm{Mb})$ of reference genomic regions in G. hirsutum have selection signals during domestication and improvement, providing a genetic resource for exploring variations controlling the change of agronomic traits. Using a limited collection of wild and landrace accessions, previous studies have not been able to 
a

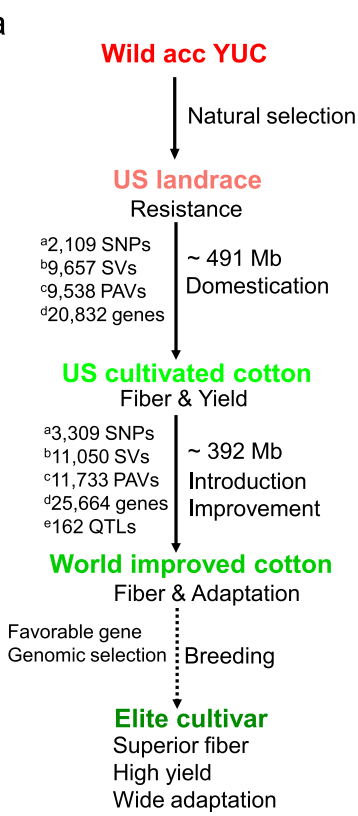

d

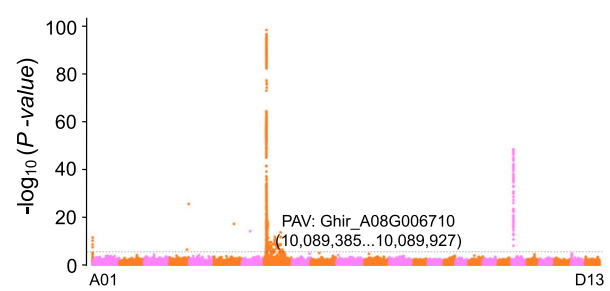

f
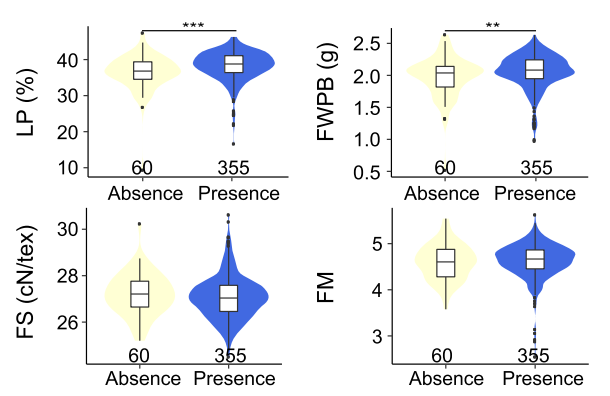

b

Landrace ImpUSO ImpCHN

$n=256 \quad n=438 \quad n=929$

C

COL2

CIP1

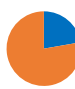

LMI1

(

GRF

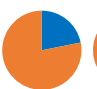

KCS2

(1)

CesA6

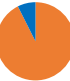

PRF3
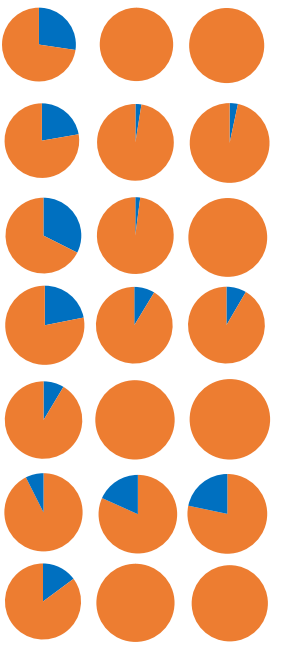

Ancestral allele

Derived allele
Landrace ImpUSO ImpCHN

$S C D$

$182 n=206 \quad n=592$

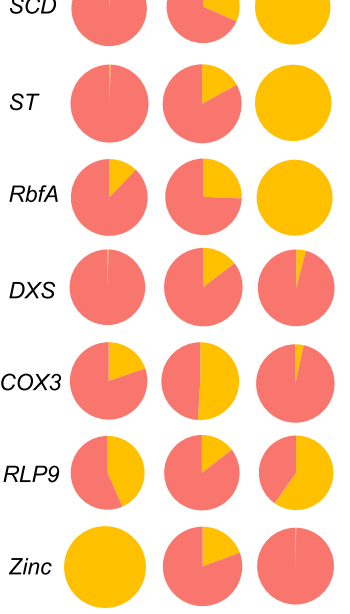

Presence allele

Absence allele

e

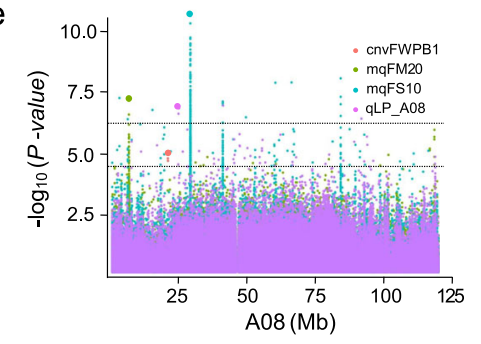

g

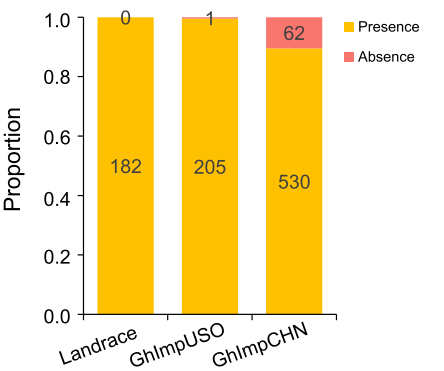

Fig. 6 An available pan-genome dataset for cotton breeding. a A four-step model of variation during cotton domestication and breeding. $\mathbf{b}$ The spectrum of gene allele frequencies at the causal SNP polymorphisms of COL2, CIP1, PRF3, LMI1, GRF, KCS2, and CesA6 in landrace and two geographic groups. c The spectrum of domesticated PAV allele frequ encies of seven genes in landrace and two geographic groups. d An example of functional PAV located on the A08 chromosome. The dashed line in Manhattan plot indicates the threshold for GWAS signals $\left(P<2.62 \times 10^{-8} ;-\log P>7.6\right)$. This locus includes four QTLs (lint percentage (LP), fiber weight per boll (FWPB), fiber micronaire (FM), fiber strength (FS)). e Four QTLS were displayed in a panel of multiple accessions. The two dashed lines represent GWAS thresholds for CNV $(-\log P>6.45)$ and SNP (-log $P>4.42)$, respectively. $\mathbf{f}$ the phenotypic difference between presence and absence groups. The numbers below the violin plots show the accession numbers. The significance difference was calculated with a two-sided Wilcoxon rank-sum test ( ${ }^{* *} P<0.001,{ }^{* *} P<0.01$ ). $g$ Presence frequencies of Ghir_A08G006710 in 182 landrace, 206 GhlmpUSO, and 592 GhlmpCHN accessions 
identify as many selection signals as possible, especially from landrace to the American cotton domestication process [3-6]. This comprehensive variome map provides a new perspective on variation landscape, QTL locations, domestication map, and potential molecular targets for cotton improvement.

Given the notion that variation identification based on mapping data against a single genome cannot fully resolve the entire variation repertoire of germplasm resources, pangenome analysis provides an ideal alternative for allele mining associated with desirable phenotypes during crop improvement. In this study, we made an attempt of pan-genome assembly using the accessible genomic data. We constructed pan-genomes for G. hirsutum and G. barbadense species using a conservative reference-guide strategy [21], which include $1041 \mathrm{Mb}$ and $309 \mathrm{Mb}$ extra sequences not captured by the reference genomes. G. hirsutum species has a higher percentage of variable genes (38\% of 39,278 genes) than G. barbadense (14\% of 11,359 genes). For Gbpan-genome, the small number of variable genes is due to the fewer number of sequenced accessions. A deep re-sequencing of a larger set of G. barbadense germplasm resources is needed to improve the resolution of pan-genome analysis. $G$. hirsutum has a considerable proportion (38\%) of variable genes as comparable to other plants, such as wild soybean [18] (51\%), Brassica oleracea [21] (19\%), bread wheat [22] (64\%), Brachypodium distachyon [23] (45\%), rice [24] (52\%), tomato [26] (26\%), sunflower [27] (25\%), sesame [28] (42\%), and Brassica napus [29] (38\%). This proportion of variable genes may be increased with deep re-sequencing data and long-read-based pan-genome assembly. Pan-genome analysis provides an opportunity for understanding of genetic diversity using gene pools to discover gene loss during domestication and improvement, and beneficial alleles and genes in wild counterparts could be used for crop improvement. In this study, PAV presence frequency analyses reveal the loss of 6753 and 3866 genes during cotton domestication and improvement, consistent with the trend found in tomato domestication [26]. We analyzed some PAVs associated with cotton agronomic traits, which allows the identification of potential causal genes (Fig. 6d). Inevitably speaking, the current pangenome assembly based on short reads leads to many iteratively assembled fragments, so more accessions need deep sequencing to allow de novo genome assembly. The annotation of non-reference genes may be combined with population transcriptome data, which can contribute to more precise annotation of coding genes and non-coding RNAs. In the future, long-read sequencing technologies will be required to integrate new genomics approaches to accurately identify structural variations and construct graphical pan-genomes [50-52].

\section{Conclusions}

In summary, our variome analyses reveal genomic landscape diversity and domestication process in allotetraploid cotton and identify some novel QTLs that may contribute to phenotypic diversity. Pan-genome analyses discover genes that have been lost during domestication and explore the possible impact of some PAVs on fiber traits. Further genetic manipulation of these QTLs and genes will advance precision breeding of this important crop.

\section{Methods}

\section{Collection of sequencing data of 1961 cotton accessions}

Genome re-sequencing data of 1874 cottons were downloaded from National Center for Biotechnology Information (NCBI) database. In this study, we sequenced $87 \mathrm{G}$. 
hirsutum and G. barbadense cultivated accessions (Additional file 1: Table S1). In total, 1961 cotton accessions were obtained, including 1655 G. hirsutum (AD) 1 , 270 G. barbadense $(\mathrm{AD})_{2}, 26$ wild Gossypium species $(\mathrm{AD})_{3}-(\mathrm{AD})_{7}$, and 10 diploid (two Gossypium arboreum $\left(\mathrm{A}_{2}\right)$, two Gossypium anomalum $\left(\mathrm{B}_{1}\right)$, one Gossypium davidsonii $\left(\mathrm{D}_{3}\right)$, two Gossypium gossypioides $\left(\mathrm{D}_{6}\right)$, three Gossypium exiguum $\left(\mathrm{K}_{1}\right)$ accessions) species [3-6, 16, 33, 34] (Additional file 1: Table S1).

\section{Identification of SNPs and InDels from 1913 accessions}

After discarding duplicated accessions, a total of 1913 cottons were used for SNPs/ InDels variation calling (Additional file 1: Table S1), including 1623 allotetraploid G. hirsutum $(\mathrm{AD})_{1}, 261 \mathrm{G}$. barbadense $(\mathrm{AD})_{2}$, and 26 wild Gossypium species $(\mathrm{AD})_{3}$ $(\mathrm{AD})_{7}$, two Gossypium arboreum $\left(\mathrm{A}_{2}\right)$-genome and one Gossypium davidsonii $\left(\mathrm{D}_{3}\right)$ species [3-6, 16, 33, 34]. Two allotetraploid reference genomes (Gossypium hirsutum acc. TM-1 and Gossypium barbadense acc. 3-79) [12] and their annotations were downloaded from CottonGen https://www.cottongen.org/. Raw pair-end reads were filtered with Trimmomatic (v0.32, MINLEN: 75) [53]. Clean reads were aligned against reference genomes using BWA-MEM (v0.7.10-r789). Duplicated mapping reads were filtered using picard-tools, and uniquely mapped reads were retained for further analysis. The reads around InDels from BWA [54] alignment were realigned by GATK (v4.0.1) [55] with RealignerTargetCreator and InDelRealigner programs with parameter setting -stand_call_conf 30. To obtain high-confidence variants, we retained the shared variants by GATK and SAMtools [56] with sequencing depth of at least 6. The scaffolds were excluded from further analysis. Finally, GVCF files were merged with "CombineGVCFs" and the missing rate was filtered by VCFtools. The missing genotypes were imputed using Beagle (v5.0) with hidden Markov model [57]. A total of 119,678,187 SNPs and 12,354,432 InDels were identified from 1913 cotton accessions. The detailed filtering processes were as follows: (1) high-quality SNPs set with $1 \% \leq$ minor allele frequency $(\mathrm{MAF}) \leq 99 \%$ and InDels with a maximum length of $20 \mathrm{bp}$ were retained. Missing rates of more than $80 \%$ were discarded in specified populations; (2) a filtered set of $\sim 25$ million SNPs from $\sim 63$ million bi-allelic SNPs was retained with MAF $\geq 0.01$; (3) core SNP set of $\sim 19$ million was obtained from $\sim 25$ million SNPs with criteria of at least five accessions having homozygote for each SNP. The core variation set has 19,246,497 SNPs and 4,815, 125 InDels which were used for further analysis (Additional file 1: Tables S2 and S3). The different sub-population SNPs were filtered with the same criteria. The core SNPs and InDels were annotated using ANNOVAR program [58].

\section{Population structure analysis of 1913 accessions}

We chose randomly 5 SNP sets (200,000 SNPs for each) from the core SNP set $(19,246$, 497) for population structure analysis. First, STRUCTURE [59] was run on $5 \times 200,000$ SNPs with $K$ ranging from 3 to 15 , and $K=12$ was determined as the optimal value by the Structure Harvest subprogram with the "evanno" method. The Q-matrices were merged using CLUMPP [60] based on the sorting of $K=12$. One random SNP set was used to construct a neighbor-joining tree with PHYLIP (v3.696) [61] with 1000 bootstrap replicates and was visualized using online tool iTOL (https://itol.embl.de/). Principal component analysis (PCA) was performed using gcta64 [62] program with MAF $\geq$ 
0.05 . The weighted Fixation statistics $(F \mathrm{st})$ and nucleotide diversity $(\pi)$ were calculated by VCFtools (v1.1.14) [63] in a 100-kb sliding window with a step size of $20 \mathrm{~kb}$.

\section{Linkage disequilibrium (LD) analysis}

Linkage disequilibrium (LD) was calculated using PLINK (v1.90b6.10) [64] with parameter settings (--ld-window-r ${ }^{2} 0$--ld-window 99999 --ld-window-kb 1000). The pairwise $r^{2}$ values were calculated by two SNPs across whole genome. The LD decay plot was shown in average $1 \mathrm{~kb}$ bins using a Perl script.

\section{Genome-wide selective sweep analysis}

We identified genomic selection and improvement signals using two strategies. For the domestication regions, we combined two major cultivated cotton groups (438 accessions from USO and other geographical regions, 929 accessions from China) into an improved group to exclude genetic drift. In total, 256 landraces and 1364 improved G. hirsutum accessions were used for domestication sweeps. Nucleotide diversity $(\pi)$ was calculated from landrace, improved GhImpUSO and GhImpCHN groups. The ratio of nucleotide diversity $\left(\pi_{\text {Landrace }} / \pi_{\text {Improved }}\right)$ for landrace versus improved cultivars was used to define candidate domestication selection regions. XP-CLR [65] (v1.0, -w1 0.005 20020001 -p0 0.9) method was used to filter candidate domestication regions. To perform XP-CLR analysis, SNPs were assigned at genetic positions according to a released genetic map [12]. The top 5\% XP-CLR values were selected. The overlapping regions in $\pi$ ratio and XP-CLR analyses were identified to be high-confidence domestication sweep regions. The adjacent DSR signals were merged. The GhImpUSO/GhImpCHN ratio was used to identify improved regions during breeding. The domesticated homeologous gene pairs were detected by the reciprocal best BLAST hit between At- and Dtsubgenomes. Syntenic blocks were detected using MCScanX [66]. The expression levels of domestication-related genes were calculated between wild/landraces and improved cultivars using data in previous studies [3, 47].

\section{Identification of structural variations}

The DELLY (v0.7.2) [67] program was used to identify structural variation (SV), integrating the strategies of read depth, read pair, and split read for SV identification. DELLY was used to identify deletions (DEL), insertions (INS), duplications (DUP), inversions (INV), and translocations (TRA) for each accession. Breakdancer (v1.3.6) [68] was also used to identify insertions, deletions, inversions, and inter- and intrachromosomal translocations for each accession according to the mapped pair-end reads with unexpected separation distance or orientation. Breakdancer-max (-q 20 -y 30) was used for SV identification of each accession. The shared breakpoints of SVs were subject to a filtering process with mapping read depth of more than 10x. The SVs in all cotton accessions were merged into a population-scale VCF file using BCFtools. For the analysis of SV genotypes, the high-quality SVs filtered as "LowQual" and "IMPRECISE" were further retained only with split-read (SR) consensus alignment of more than 3 and the length of more than $50 \mathrm{bp}$ and less than $1 \mathrm{Mb}$. Two adjacent SVs were combined as a single SV if the distance between start coordinate of one SV and end 
coordinate of the other SV was less than $500 \mathrm{bp}$, and the overlapping region occupied more than $50 \%$ of the total size.

\section{Identification of CNVs}

The copy number variations (CNVs) were detected using CNVcaller [69]. Briefly, the reference genome was split into 800 bp overlapping sliding windows. Second, we generated the reference genome index and processed the BAM file of each accession. The boundaries of CNV regions (CNVR) were detected using normalized mean read depth (RD). The CNV minimum frequency of gain/loss individuals (-f 0.05), homozygotes (-h 3 ), and RD of adjacent windows are significantly correlated (-r 0.5). At last, the CNV genotype were clustered with the input sample using a Gaussian Mixture Model. The minor allele frequency of 0.01 was used in each specific population.

\section{Meta-genome-wide association study for fiber and agronomic traits}

We performed a genome-wide association study on three independent experiments for fiber length (FL), fiber strength (FS), fiber micronaire (FM), fiber elongation (FE), length uniformity (FU), boll weight (BW), lint percentage (LP), seed index (SI), lint index (LI), fiber weight per boll (FWPB), and flowering date (FD), using re-sequencing data of 267 accessions from Huazhong Agricultural University (HZAU) [3, 14], 263 accessions from Nanjing Agricultural University (NJAU) [5], and 419 accessions from Hebei Agricultural University (HBAU) [6]. After discarding accessions with missing phenotypes, a total of 264, 207, and 419 accessions from the HZAU, NJAU, and HZAU were retained, respectively. We merged best linear unbiased prediction (BLUP) [70] values of 890 non-redundant accessions in three independent experiments to conduct Meta-GWAS. The 2,787,330, 677,013, 2,371,414 and 2,291,437 high-quality SNPs (MAF $\geq 0.05$ and homozygote more than five accessions) were used for GWAS analysis in 264,207,419,890 accessions using the TASSEL5.0 [71] with a mixed linear model $(\mathrm{P}+\mathrm{G}+\mathrm{Q}+\mathrm{K})$ and FastLMM [72], respectively. The significant threshold was set as $1 /$ $N$ (independent case), and $0.05 / N$ (Meta-GWAS) as filtering parameter (" $N$ " represents the total number of SNPs). For the CNV-based GWAS, we used 26,831 CNVs identified from 419 G. hirsutum accessions released by the HBAU (MAF $\geq 0.05)$ to identify CNV-based QTLs.

\section{Pan-genome construction based on short reads}

The unaligned reads were extracted using SAMtools with "-b -f 4" and "-f 68 -F 8 and -f 132 -F 8." We assembled all unmapped paired reads and unpaired single reads for each accession with MaSuRCA (v3.2.1) [73] assembler (cgwErrorRate $=0.15, \mathrm{PE}=$ "PE 300 50," LIMIT_JUMP_COVERAGE = 300, KMER_COUNT_THRESHOLD =1). The initial contigs with a length of longer than $500 \mathrm{bp}$ were retained. The long contigs were aligned against reference genome using nucmer (-c 90 -1 40) program in MUMmer (v4.0.0) package [74]. The redundant sequences were filtered using CD-HIT (v4.8.1) [75] with command "-c 0.9 -G 0 -aL 0.90 -AL 500 -aS 0.9 -T 0 -M 1500000." The contig filtering steps are as follows: (1) remaining sequences from cotton chloroplast genome (GenBank: DQ345959) and mitochondrial genome (GenBank: JX944505.1) were identified using BLASTN and MUMmer package with nucmer "-1 90." To ensure that 
contigs of each accession were absent from the reference genome, we aligned these contigs against the reference genome; (2) unaligned contigs from the archaea, bacteria, and viral genomes (Jun 18, 2019) were discarded using the Kraken (v2) [76]; (3) nonredundant contigs were used to search the NCBI nt database (20171030) using BLAST (-e 1e-05; -best_hit_overhang 0.25 -perc_identity 0.8 ; -max_target_seqs 10 ) to identify other contaminants; (4) remaining contigs were subject to an all-versus-all alignment with nucmer and BLASTN (-e 1e-05, -b 200 -v 200) to ensure the non-redundancy. Contig sequences with a similarity of $90 \%$ and a length of $90 \%$ were filtered out among the cultivars. The non-reference sequences that were not aligned to sequences of higher plants in NCBI nt database were considered as contaminant sequences, according to previous rice pan-genome [24]. The Ghpan-genome sequences were generated by combining the $2347 \mathrm{Mb}$ of "TM-1" reference sequences and $1041 \mathrm{Mb}$ of final non-reference sequences. The Gbpan-genome sequences consisted of $2266 \mathrm{Mb}$ of "3-79" reference sequence and $309 \mathrm{Mb}$ of final non-reference sequences.

\section{PacBio sequencing and de novo assembly of 10 representative cotton accessions}

According to phylogenetic tree, three wild/landrace accessions, three GhImpUSO, and four GhImpCHN cultivars of G. hirsutum for different sub-population were used for evaluating Ghpan-genome coverage. Genomic DNA was extracted from young leaves using $\mathrm{CTAB}$ method. The PacBio library was constructed and sequenced on PacBio Sequel platform. Long reads were assembled using MECAT (ErrorRate $=0.02$ ) assembler [77]. The 70x depth Illumina pair-end reads were used to polish the PacBio assembly using pilon (v1.23) program [78]. After two rounds of polishing, 2,550,224 SNPs, 13, 154,090 insertions, and 2,151,774 deletions were corrected on average for each cotton accession. The assemblies of wild accessions, landraces, and modern cultivated cotton were subject to assessment of assembly completeness using BUSCO (v3.1.0) [79] with embryophyta_odb9 database as a reference. This showed that 1369 (95.1\%), 1380 (95.8\%), and 1374 (95.4\%) integrity BUSCO hits were found for landrace, GhImpUSO, and GhImpCHN groups, respectively (Additional file 2: Figure S18b).

\section{Annotation of pan-genome genes}

We used de novo and homology-based prediction of non-reference genes (only contigs with a length of more than $1000 \mathrm{bp}$ were used for gene prediction). First, RepeatModeler (v1.0.11) (http://www.repeatmasker.org/RepeatModeler/) was used for de novo construction of repeat library in the non-reference genome, and the repeat sequences were masked by RepeatMask (v4.0.7) [80]. The protein-coding genes were predicted in nonreference genomes with MAKER2 pipeline [81]. Gene prediction included ab initio prediction and protein homology-based prediction. For ab initio gene prediction, AUGUSTUS (v3.3.1) [82] and SNAP (v2006-07-28) [83] were trained for two rounds by MAKER. Cotton expressed sequence tags (ESTs, MAY 2019) were downloaded from NCBI and aligned against the non-reference sequences using BLASTN. Cotton protein sequences were downloaded from NCBI and UniProtKB databases and were aligned against the non-reference sequences with BLASTX. We excluded non-reference genes with less than $500 \mathrm{bp}$ on both sides of contigs. These transcripts were aligned to reference transcripts to remove potential redundant transcripts. These non-reference 
transcripts were also subjected to all-by-all alignment. The final protein sequences translated from transcripts were aligned using InterProScan (v5) [84]. Transcripts with at least one evidence (Interpro, Pfam, GO, KEGG) supporting annotation were retained.

For the functional annotation of non-reference genes, protein sequences were aligned against the NCBI non-redundant (nr) and InterProScan (v5). GO enrichment analysis of core and variable genes were performed for Ghpan-genome and Gbpan-genome using Fisher's exact test method.

\section{Gene presence/absence variation (PAV) analysis}

First, the raw reads from each accession were aligned to the pan-genome sequences using BWA-MEM with default parameters. The PAVs were detected by SGSGeneLoss [85] (v1.0) with at least two covered reads $(\operatorname{minCov}=2$, lostCutoff $=0.2)$. If more than $80 \%$ of exon regions were covered, this gene was called present with the " $1 / 1$ " genotype. We defined variable genes and divided them into three categories: softcore, shell, and cloud genes. For the G. hirsutum pan-genome, the softcore, shell, and cloud genes were present in $97-100 \%, 1-97 \%$, and less than $1 \%$ of the accessions in specified population, respectively. For G. barbadense pan-genome, the softcore, shell, and cloud genes were present in $98-100 \%, 3-97 \%$, and less than $2 \%$ of the accessions in specified population, respectively. The $K_{a}$ and $K_{s}$ values were calculated to estimate evolutionary rate by KaKs_Calculator (v2.0) [86] with multiple alignments of core, softcore, shell, and cloud genes using MAFFT (v7.453) [87]. The shell and cloud genes were combined as flexible genes. For the PAV population analysis, we selected PAV genes present in specified population and discarded un-mapping non-reference genes. The phylogenetic tree was constructed using IQ-TREE [88] program based on the binary flexible PAV genes with 1000 bootstraps according to a maximum-likelihood method. The pan-genome saturation curve analysis was repeated for 1000 random combinations with five replicates of cotton genome orders starting with two and ending with 1020 of G. hirsutum accessions, and 177 of G. barbadense accessions.

\section{Selection of PAVs during cotton domestication and improvement}

To identify PAVs undergoing selection during cotton domestication and improvement, the PAV presence frequencies were calculated in two groups (landrace versus Improved USO and Improved CHN for domestication; GhImpUSO versus GhImpCHN for improvement). The significantly different PAV frequency for each gene between domestication and improvement groups was calculated using Fisher's exact test. The $P$ value was determined in all PAVs and was then corrected via false discovery rate (FDR). PAVs with significantly different frequencies $(F D R<0.001$ and Ghlandrace/GhImproved fold change $>2$ defined as "unfavorable" or fold change $<0.5$ "favorable") were identified as those with domestication/improvement selection signals. The continuously selected genes in landrace, GhImpUSO, and GhImpCHN sub-population were defined as "favorable gain" (gene presence frequency: GhImpCHN > GhImpUSO > landrace), "favorable loss" (GhImpUSO > landrace and GhImpCHN < GhImpUSO), "unfavorable gain" (landrace > GhImpUSO and GhImpCHN > GhImpUSO), and "unfavorable loss" (GhImpCHN < GhImpUSO < landrace). 


\section{Identification of PAV-associated SNPs}

To associate SNPs with PAV genes, we analyzed linkage disequilibrium between PAVs and SNPs using FastLMM [72]. According to the G. hirsutum PAV analysis, 1196 PAVs (MAF $\geq 0.02$ ) were used for genome-wide association analysis in 415 accessions (exclude abnormal samples) [6]. The PAV presence and absence served as the "phenotype, " and 1,904,926 SNPs served as "genotype" according to a previous study [27]. To control the false-positive rate of significant hits, we used a threshold of $0.05 / N$ to filter association peaks. The significant PAV loci that overlapped with trait-QTLs were considered to be associated with agronomic traits, and the location of QTLs represented the positions of non-reference PAVs. Reference PAV-associated trait-SNPs were selected manually.

\section{CRISPR/Cas9 mutagenesis experiment}

Computational sgRNA design for Ghir_D05G013680 gene and vector construction were described in a previous study [89]. Gossypium hirsutum cultivar accession Jin668 was used for Agrobacterium-mediated transformation as described in our previous study [90]. The transgenic cotton plants were confirmed by genotyping polymerase chain reaction (PCR), and then positive individual was used for Hi-TOM target sequencing [91]. The T0 transgenic positive plants were transplanted in the greenhouse, in order to harvest T0 seeds. The T1 generation plants were cultivated in the experimental field of Huazhong Agricultural university. The edited T1 transgenic line was validated by Sanger sequencing. The fiber quality-related traits were measured with 10 cotton bolls from T1 and wild type plants by a High-Volume Instrument (HVI) (HFT9000, Premier, India).

\section{Phytohormone measurement}

About 150-mg fiber samples were extracted twice with cold methanol $(80 \%$ [v/v]) by sharking overnight at $4{ }^{\circ} \mathrm{C}$ with three biological replicates. Indole-3-acetic-2,2- $\mathrm{d} 2$ acid (IAA; Sigma-Aldrich), ${ }^{2} \mathrm{H}_{6}$-abscisic acid (ABA; Olchemim), and ( \pm ) 9,10-dihydro-jasmonic acid (JA; Olchemim) were added to each sample as an internal standard. The quantification of IAA, ABA, JA, and jasmonoyl-isoleucine (JA-Ile) was performed on an ABI 4000 Q-Trap system (Applied Biosystems) according to a method described previously [92].

\section{Supplementary Information}

Supplementary information accompanies this paper at https://doi.org/10.1186/s13059-021-02351-W.

Additional file 1: Table S1. Summary of genomic sequencing data of 1961 cottons in this study. Table S2. Summary of SNP filtering in 1913 G. hirsutum, G. barbadense and other Gossypium species accessions for each chromosome. Table S3. Summary of InDels filtering in 1913 accessions for each chromosome. Table S4. Summary of SNP filtering in $1623 \mathrm{G}$. hirsutum accessions for each chromosome. Table S5. Summary of SNP and InDel filtering in 261 G. barbadense accessions for each chromosome. Table S6. The SNP and InDel annotation of subpopulation. Table S7. The number of structural variations filtered by four steps in each subpopulation. Table S8. Summary of copy number variations for each chromosome in different subpopulation. Table S9. The SNP-based domestication sweeps and covering genes. Table S10. The SNP-based improvement signals and covering genes. Table S11. Continuously selected signals and genes during domestication and improvement. Table S12. The CNV-based domestication signals and overlapped SNP-based signals. Table S13. The CNV-based improvement signals and overlapped SNP-based signals. Table S14. Significant SNP-based GWAS signals for 15 agronomic traits. Table S15. Summary of snpQTLs that overlapped with domestication and improvement signals. Table S16. Summary of pleiotropic snpQTLs in multiple panels. Table S17. Summary of pleiotropic cnvQTLs in 419 panel accessions. Table S18. Summary of cnvQTLs that overlap with domestication and improvement signals. Table S19. Summary of assembly non- 
reference contigs between G. hirsutum and G. barbadense. Table S20. Filtering non-reference sequences following several filtering steps. Table S21. Protein-coding genes predicted in the $G$. hirsutum non-reference genome. Table S22. Protein-coding genes predicted in the G. barbadense non-reference genome. Table S23. Summary of PacBio reads and Illumina reads for 10 representative G. hirsutum cotton accessions. Table S24. Summary of polished contigs for 10 representative $G$. hirsutum accessions and draft genomes mapped to the GhPangenome. Table S25. Meta-genome likely assembly for landrace, American and modern cotton variety groups. Table S26. Selected PAVs during cotton domestication. Table S27. Selected PAVs during cotton improvement. Table S28. Selected PAVs during both domestication and improvement. Table S29. Summary of selective regions and covering genes during domestication and improvement. Table S30. Detail information of functional SNP variation in landrace and two geographic groups. Table S31. Summary of PAVs associated with QTLS.

Additional file 2. Figures S1-S30.

Additional file 3. Supplementary Notes.

Additional file 4. Review history.

\section{Acknowledgements}

We are very grateful to Professor J. Udall (USDA) for releasing the sequencing data of wild and landrace accessions. We thank Z. Ma (Hebei Agricultural University) for sharing phenotype data of 419 cotton accessions. We thank the mid-term germplasm bank of China Cotton Research Institute for providing wild cotton accessions. We also thank all the germplasm providers. We thank the high-performance computing platform at National Key Laboratory of Crop Genetic Improvement in Huazhong Agricultural University.

\section{Peer review information}

Kevin Pang was the primary editor on this article and managed its editorial process and peer review in collaboration with the rest of the editorial team.

\section{Review history}

The review history is available as Additional file 4

\section{Authors' contributions}

M.W., S.J., and X.Z. conceived and managed the project. J.L. collected all datasets and conducted PacBio and Illumina sequencing. J.L., Z.L., H.S., Z.X., Y.M., B.Z., and L.P. performed variome analyses and panel GWAS. J.L., P.W., Q.W., and M.S. performed gene functional analysis. J.L. performed de novo genome assembly, pan-genome construction, and PAV selection. M.W., J.L., D.Y., L.T., L.Z., L-L.C., and K.L. contributed to project discussion and interpreted the results. J.L. prepared the figures and wrote the manuscript draft, and M.W., X.Z., K.L., and S.J. revised it. The author(s) read and approved the final manuscript.

\section{Funding}

This project was supported by funding from the National Natural Science Foundation of China $(31922069,31801405)$ to M.W and National Natural Science Foundation of China (31830062) to X.Z. This work was also funded by China Postdoctoral Science Foundation (2019 M662663) and the National Natural Science Foundation of China (32001595) to J.L.

\section{Availability of data and materials}

The short reads of 87 cotton accessions and PacBio long reads of 10 representative G. hirsutum accessions have been submitted to NCBI under the accession number PRJNA576032 (https://www.ncbi.nlm.nih.gov/bioproject/PRJNA576032, [93]. The variome data set (SNPs, InDels, CNVs, PAVs), non-reference sequences, annotated genes of G. hirsutum and G. barbadense species, and other source data have been deposited in Figshare database (https://figshare.com/s/cb3c104 782a1dcd90ab0) [94]

\section{Declarations}

Ethics approval and consent to participate

Not applicable.

\section{Consent for publication}

Not applicable.

\section{Competing interests}

The authors declare no conflict of interest.

\section{Author details}

${ }^{1}$ National Key Laboratory of Crop Genetic Improvement, Huazhong Agricultural University, Wuhan, China. ${ }^{2}$ College of Plant Science and Technology, Huazhong Agricultural University, Wuhan, China. ${ }^{3}$ Hubei Key Laboratory of Agricultural Bioinformatics, College of Informatics, Huazhong Agricultural University, Wuhan, China. ${ }^{4}$ Department of Biosciences, Durham University, Durham, UK. 
Received: 29 September 2020 Accepted: 14 April 2021

Published online: 23 April 2021

\section{References}

1. Wendel JF. New World tetraploid cottons contain Old-World cytoplasm. Proc Natl Acad Sci U S A. 1989;86(11):4132-6. https://doi.org/10.1073/pnas.86.11.4132.

2. Senchina DS. Rate variation among nuclear nenes and the age of polyploidy in Gossypium. Mol Biol Evol. 2003;20(4): 633-43. https://doi.org/10.1093/molbev/msg065.

3. Wang M, Tu L, Lin M, Lin Z, Wang P, Yang Q, Ye Z, Shen C, Li J, Zhang L, Zhou X, Nie X, Li Z, Guo K, Ma Y, Huang C, Jin S, Zhu L, Yang X, Min L, Yuan D, Zhang Q, Lindsey K, Zhang X. Asymmetric subgenome selection and cis-regulatory divergence during cotton domestication. Nat Genet. 2017;49(4):579-87. https://doi.org/10.1038/ng.3807.

4. Fang L, Gong H, Hu Y, Liu C, Zhou B, Huang T, Wang Y, Chen S, Fang DD, Du X, et al. Genomic insights into divergence and dual domestication of cultivated allotetraploid cottons. Genome Biol. 2017;18(1):33. https://doi.org/10.1186/s13059017-1167-5.

5. Fang L, Wang Q, Hu Y, Jia Y, Chen J, Liu B, Zhang Z, Guan X, Chen S, Zhou B, Mei G, Sun J, Pan Z, He S, Xiao S, Shi W, Gong W, Liu J, Ma J, Cai C, Zhu X, Guo W, du X, Zhang T. Genomic analyses in cotton identify signatures of selection and loci associated with fiber quality and yield traits. Nat Genet. 2017:49(7):1089-98. https://doi.org/10.1038/ng.3887.

6. Ma Z, He S, Wang X, Sun J, Zhang Y, Zhang G, Wu L, Li Z, Liu Z, Sun G, Yan Y, Jia Y, Yang J, Pan Z, Gu Q, Li X, Sun Z, Dai P, Liu Z, Gong W, Wu J, Wang M, Liu H, Feng K, Ke H, Wang J, Lan H, Wang G, Peng J, Wang N, Wang L, Pang B, Peng Z, Li R, Tian S, Du X. Resequencing a core collection of upland cotton identifies genomic variation and loci influencing fiber quality and yield. Nat Genet. 2018;50(6):803-13. https://doi.org/10.1038/s41588-018-0119-7.

7. Westengen OT, Huaman Z, Heun M. Genetic diversity and geographic pattern in early south American cotton domestication. Theor Appl Genet. 2005;110(2):392-402. https://doi.org/10.1007/s00122-004-1850-2.

8. Lacape JM, Dessauw D, Rajab M, Noyer JL, Hau B. Microsatellite diversity in tetraploid Gossypium germplasm: assembling a highly informative genotyping set of cotton SSRs. Mol Breed. 2006;19(1):45-58. https://doi.org/10.1007/s11032-0069042-1.

9. Tyagi P, Gore MA, Bowman DT, Campbell BT, Udall JA, Kuraparthy V. Genetic diversity and population structure in the US upland cotton (Gossypium hirsutum L.). Theor Appl Genet. 2014;127(2):283-95. https://doi.org/10.1007/s00122013-2217-3.

10. Hinze LL, Gazave E, Gore MA, Fang DD, Scheffler BE, Yu JZ, Jones DC, Frelichowski J, Percy RG. Genetic diversity of the two commercial tetraploid cotton species in the Gossypium diversity Reference Set. J Hered. 2016;107(3):274-86. https:// doi.org/10.1093/jhered/esw004.

11. Percy RG, Wendel JF. Allozyme evidence for the origin and diversification of Gossypium barbadense L. Theor Appl Genet. 1990;79(4):529-42. https://doi.org/10.1007/BF00226164.

12. Wang M, Tu L, Yuan D, Zhu D, Shen C, Li J, Liu F, Pei L, Wang P, Zhao G, Ye Z, Huang H, Yan F, Ma Y, Zhang L, Liu M, You J, Yang Y, Liu Z, Huang F, Li B, Qiu P, Zhang Q, Zhu L, Jin S, Yang X, Min L, Li G, Chen LL, Zheng H, Lindsey K, Lin Z, Udall JA, Zhang X. Reference genome sequences of two cultivated allotetraploid cottons, Gossypium hirsutum and Gossypium barbadense. Nat Genet. 2019;51(2):224-9. https://doi.org/10.1038/s41588-018-0282-X.

13. Hu Y, Chen J, Fang L, Zhang Z, Ma W, Niu Y, Ju L, Deng J, Zhao T, Lian J, Baruch K, Fang D, Liu X, Ruan YL, Rahman MU, Han J, Wang K, Wang Q, Wu H, Mei G, Zang Y, Han Z, Xu C, Shen W, Yang D, Si Z, Dai F, Zou L, Huang F, Bai Y, Zhang Y, Brodt A, Ben-Hamo H, Zhu X, Zhou B, Guan X, Zhu S, Chen X, Zhang T. Gossypium barbadense and Gossypium hirsutum genomes provide insights into the origin and evolution of allotetraploid cotton. Nat Genet. 2019;51(4):739-48. https:// doi.org/10.1038/s41588-019-0371-5.

14. Huang C, Nie X, Shen C, You C, Li W, Zhao W, Zhang X, Lin Z. Population structure and genetic basis of the agronomic traits of upland cotton in China revealed by a genome-wide association study using high-density SNPs. Plant Biotechnol J. 2017;15(11):1374-86. https://doi.org/10.1111/pbi.12722.

15. Li Z, Wang P, You C, Yu J, Zhang X, Yan F, Ye Z, Shen C, Li B, Guo K, Liu N, Thyssen GN, Fang DD, Lindsey K, Zhang X, Wang M, Tu L. Combined GWAS and eQTL analysis uncovers a genetic regulatory network orchestrating the initiation of secondary cell wall development in cotton. New Phytol. 2020;226(6):1738-52. https://doi.org/10.1111/nph.16468.

16. Wen T, Wu M, Shen C, Gao B, Zhu, Zhang X, You C, Lin Z. Linkage and association mapping reveals the genetic basis of brown fibre (Gossypium hirsutum). Plant Biotechnol J. 2018;16(9):1654-66. https://doi.org/10.1111/pbi.12902.

17. Tettelin H, Masignani V, Cieslewicz MJ, Donati C, Medini D, Ward NL, Angiuoli SV, Crabtree J, Jones AL, Durkin AS, DeBoy RT, Davidsen TM, Mora M, Scarselli M, Margarit y Ros I, Peterson JD, Hauser CR, Sundaram JP, Nelson WC, Madupu R, Brinkac LM, Dodson RJ, Rosovitz MJ, Sullivan SA, Daugherty SC, Haft DH, Selengut J, Gwinn ML, Zhou L, Zafar N, Khouri H, Radune D, Dimitrov G, Watkins K, O'Connor KIB, Smith S, Utterback TR, White O, Rubens CE, Grandi G, Madoff LC, Kasper DL, Telford JL, Wessels MR, Rappuoli R, Fraser CM. Genome analysis of multiple pathogenic isolates of Streptococcus agalactiae: implications for the microbial "pan-genome". Proc Natl Acad Sci U S A. 2005;102(39):13950-5. https://doi.org/10.1073/pnas.0506758102.

18. Li YH, Zhou G, Ma J, Jiang W, Jin LG, Zhang Z, Guo Y, Zhang J, Sui Y, Zheng L, Zhang SS, Zuo Q, Shi XH, Li YF, Zhang WK, Hu Y, Kong G, Hong HL, Tan B, Song J, Liu ZX, Wang Y, Ruan H, Yeung CKL, Liu J, Wang H, Zhang L, Guan RX, Wang KJ, Li WB, Chen SY, Chang RZ, Jiang Z, Jackson SA, Li R, Qiu LJ. De novo assembly of soybean wild relatives for pan-genome analysis of diversity and agronomic traits. Nat Biotechnol. 2014;32(10):1045-52. https://doi.org/10.1038/ nbt.2979.

19. Lu F, Romay MC, Glaubitz JC, Bradbury PJ, Elshire RJ, Wang T, Li Y, Semagn K, Zhang X, Hernandez AG, et al. Highresolution genetic mapping of maize pan-genome sequence anchors. Nat Commun. 2015;6(1):6914. https://doi.org/10.1 038/ncomms7914.

20. Yao W, Li G, Zhao H, Wang G, Lian X, Xie W. Exploring the rice dispensable genome using a metagenome-like assembly strategy. Genome Biol. 2015;16(1):187. https://doi.org/10.1186/s13059-015-0757-3.

21. Golicz AA, Bayer PE, Barker GC, Edger PP, Kim H, Martinez PA, Chan CK, Severn-Ellis A, McCombie WR, Parkin IA, et al. The pangenome of an agronomically important crop plant Brassica oleracea. Nat Commun. 2016;7(1):13390. https://doi. org/10.1038/ncomms13390. 
22. Montenegro JD, Golicz AA, Bayer PE, Hurgobin B, Lee H, Chan CK, Visendi P, Lai K, Dolezel J, Batley J, Edwards D. The pangenome of hexaploid bread wheat. Plant J. 2017;90(5):1007-13. https://doi.org/10.1111/tpj.13515.

23. Gordon SP, Contreras-Moreira B, Woods DP, Des Marais DL, Burgess D, Shu S, Stritt C, Roulin AC, Schackwitz W, Tyler L, Martin J, Lipzen A, Dochy N, Phillips J, Barry K, Geuten K, Budak H, Juenger TE, Amasino R, Caicedo AL, Goodstein D, Davidson P, Mur LAJ, Figueroa M, Freeling M, Catalan P, Vogel JP. Extensive gene content variation in the Brachypodium distachyon pan-genome correlates with population structure. Nat Commun. 2017;8(1):2184. https://doi.org/10.1038/s414 67-017-02292-8.

24. Wang W, Mauleon R, Hu Z, Chebotarov D, Tai S, Wu Z, Li M, Zheng T, Fuentes RR, Zhang F, Mansueto L, Copetti D, Sanciangco M, Palis KC, Xu J, Sun C, Fu B, Zhang H, Gao Y, Zhao X, Shen F, Cui X, Yu H, Li Z, Chen M, Detras J, Zhou Y, Zhang X, Zhao Y, Kudrna D, Wang C, Li R, Jia B, Lu J, He X, Dong Z, Xu J, Li Y, Wang M, Shi J, Li J, Zhang D, Lee S, Hu W, Poliakov A, Dubchak I, Ulat VJ, Borja FN, Mendoza JR, Ali J, Li J, Gao Q, Niu Y, Yue Z, Naredo MEB, Talag J, Wang X, Li J, Fang X, Yin Y, Glaszmann JC, Zhang J, Li J, Hamilton RS, Wing RA, Ruan J, Zhang G, Wei C, Alexandrov N, McNally KL, Li Z, Leung H. Genomic variation in 3,010 diverse accessions of Asian cultivated rice. Nature. 2018;557(7703):43-9. https:// doi.org/10.1038/s41586-018-0063-9.

25. Zhao Q, Feng Q, Lu H, Li Y, Wang A, Tian Q, Zhan Q, Lu Y, Zhang L, Huang T, Wang Y, Fan D, Zhao Y, Wang Z, Zhou C, Chen J, Zhu C, Li W, Weng Q, Xu Q, Wang ZX, Wei X, Han B, Huang X. Pan-genome analysis highlights the extent of genomic variation in cultivated and wild rice. Nat Genet. 2018;50(2):278-84. https://doi.org/10.1038/s41588-018-0041-z.

26. Gao L, Gonda I, Sun H, Ma Q, Bao K, Tieman DM, Burzynski-Chang EA, Fish TL, Stromberg KA, Sacks GL, Thannhauser TW, Foolad MR, Diez MJ, Blanca J, Canizares J, Xu Y, van der Knaap E, Huang S, Klee HJ, Giovannoni JJ, Fei Z. The tomato pan-genome uncovers new genes and a rare allele regulating fruit flavor. Nat Genet. 2019;51(6):1044-51. https://doi. org/10.1038/s41588-019-0410-2.

27. Hubner S, Bercovich N, Todesco M, Mandel JR, Odenheimer J, Ziegler E, Lee JS, Baute GJ, Owens GL, Grassa CJ, et al. Sunflower pan-genome analysis shows that hybridization altered gene content and disease resistance. Nat Plants. 2019; 5(1):54-62. https://doi.org/10.1038/s41477-018-0329-0.

28. Yu J, Golicz AA, Lu K, Dossa K, Zhang Y, Chen J, Wang L, You J, Fan D, Edwards D, Zhang X. Insight into the evolution and functional characteristics of the pan-genome assembly from sesame landraces and modern cultivars. Plant Biotechnol J. 2019;17(5):881-92. https://doi.org/10.1111/pbi.13022.

29. Dolatabadian A, Bayer PE, Tirnaz S, Hurgobin B, Edwards D, Batley J. Characterization of disease resistance genes in the Brassica napus pangenome reveals significant structural variation. Plant Biotechnol J. 2020;18(4):969-82. https://doi.org/1 $0.1111 /$ pbi.13262.

30. Liu Y, Du H, Li P, Shen Y, Peng H, Liu S, Zhou G-A, Zhang H, Liu Z, Shi M, et al. Pan-genome of wild and cultivated soybeans. Cell. 2020;182(1):162-76. https://doi.org/10.1016/j.cell.2020.05.023.

31. Song J-M, Guan Z, Hu J, Guo C, Yang Z, Wang S, Liu D, Wang B, Lu S, Zhou R, Xie WZ, Cheng Y, Zhang Y, Liu K, Yang QY, Chen LL, Guo L. Eight high-quality genomes reveal pan-genome architecture and ecotype differentiation of Brassica napus. Nature Plants. 2020;6(1):34-45. https://doi.org/10.1038/s41477-019-0577-7.

32. Lye ZN, Purugganan MD. Copy number variation in domestication. Trends Plant Sci. 2019;24(4):352-65. https://doi.org/1 0.1016/..tplants.2019.01.003.

33. Page JT, Liechty ZS, Alexander RH, Clemons K, Hulse-Kemp AM, Ashrafi H, Van Deynze A, Stelly DM, Udall JA. DNA sequence evolution and rare homoeologous conversion in tetraploid cotton. PLoS Genet. 2016;12(5):e1006012. https:// doi.org/10.1371/journal.pgen.1006012.

34. Yuan D, Grover CE, Hu G, Pan M, Miller ER, Conover JL, Hunt SP, Udall JA, Wendel JF. Parallel and intertwining threads of domestication in allopolyploid cotton. Adv Sci. 2021. https://doi.org/10.1002/advs.202003634.

35. Huang X, Kurata N, Wei X, Wang Z-X, Wang A, Zhao Q, Zhao Y, Liu K, Lu H, Li W, Guo Y, Lu Y, Zhou C, Fan D, Weng Q, Zhu C, Huang T, Zhang L, Wang Y, Feng L, Furuumi H, Kubo T, Miyabayashi T, Yuan X, Xu Q, Dong G, Zhan Q, Li C, Fujiyama A, Toyoda A, Lu T, Feng Q, Qian Q, Li J, Han B. A map of rice genome variation reveals the origin of cultivated rice. Nature. 2012;490(7421):497-501. https://doi.org/10.1038/nature11532.

36. Lin T, Zhu G, Zhang J, Xu X, Yu Q, Zheng Z, Zhang Z, Lun Y, Li S, Wang X, Huang Z, Li J, Zhang C, Wang T, Zhang Y, Wang A, Zhang Y, Lin K, Li C, Xiong G, Xue Y, Mazzucato A, Causse M, Fei Z, Giovannoni JJ, Chetelat RT, Zamir D, Städler T, Li J, Ye Z, du Y, Huang S. Genomic analyses provide insights into the history of tomato breeding. Nat Genet. 2014; 46(11):1220-6. https://doi.org/10.1038/ng.3117.

37. Zhou Z, Jiang Y, Wang Z, Gou Z, Lyu J, Li W, Yu Y, Shu L, Zhao Y, Ma Y, Fang C, Shen Y, Liu T, Li C, Li Q, Wu M, Wang M, Wu Y, Dong Y, Wan W, Wang X, Ding Z, Gao Y, Xiang H, Zhu B, Lee SH, Wang W, Tian Z. Resequencing 302 wild and cultivated accessions identifies genes related to domestication and improvement in soybean. Nat Biotechnol. 2015; 33(4):408-14. https://doi.org/10.1038/nbt.3096.

38. Huang G, Huang JQ, Chen XY, Zhu YX. Recent advances and future perspectives in cotton research. Annu Rev Plant Biol. $2021 ; 72: 2.1-2.26$.

39. Shi YH, Zhu SW, Mao XZ, Feng JX, Qin YM, Zhang L, Cheng J, Wei LP, Wang ZY, Zhu YX. Transcriptome profiling, molecular biological, and physiological studies reveal a major role for ethylene in cotton fiber cell elongation. Plant Cell. 2006;18(3):651-64. https://doi.org/10.1105/tpc.105.040303.

40. Liu R, Gong J, Xiao X, Zhang Z, Li J, Liu A, Lu Q, Shang H, Shi Y, Ge Q, lqbal MS, Deng X, Li S, Pan J, Duan L, Zhang Q, Jiang X, Zou X, Hafeez A, Chen Q, Geng H, Gong W, Yuan Y. GWAS analysis and QTL identification of fiber quality traits and yield components in upland cotton using enriched high-density SNP markers. Front Plant Sci. 2018;9:1067. https:// doi.org/10.3389/fpls.2018.01067.

41. Thyssen GN, Jenkins JN, McCarty JC, Zeng L, Campbell BT, Delhom CD, Islam MS, Li P, Jones DC, Condon BD, Fang DD. Whole genome sequencing of a MAGIC population identified genomic loci and candidate genes for major fiber quality traits in upland cotton (Gossypium hirsutum L.). Theor Appl Genet. 2019;132:989-99.

42. Song Q, Zhang T, Stelly DM, Chen ZJ. Epigenomic and functional analyses reveal roles of epialleles in the loss of photoperiod sensitivity during domestication of allotetraploid cottons. Genome Biol. 2017;18(1):99. https://doi.org/10.11 86/s13059-017-1229-8.

43. Andres RJ, Coneva V, Frank MH, Tuttle JR, Samayoa LF, Han S-W, Kaur B, Zhu L, Fang H, Bowman DT, Rojas-Pierce M, Haigler CH, Jones DC, Holland JB, Chitwood DH, Kuraparthy V. Modifications to a LATE MERISTEM IDENTITY1 gene are 
responsible for the major leaf shapes of upland cotton (Gossypium hirsutum L.). Proc Natl Acad Sci U S A. 2017;114(1): E57-66. https://doi.org/10.1073/pnas.1613593114.

44. Ma D, Hu Y, Yang C, Liu B, Fang L, Wan Q, Liang W, Mei G, Wang L, Wang H, Ding L, Dong C, Pan M, Chen J, Wang S, Chen S, Cai C, Zhu X, Guan X, Zhou B, Zhu S, Wang J, Guo W, Chen X, Zhang T. Genetic basis for glandular trichome formation in cotton. Nat Commun. 2016;7(1):10456. https://doi.org/10.1038/ncomms10456.

45. Xiao GH, Wang K, Huang G, Zhu YX. Genome-scale analysis of the cotton KCS gene family revealed a binary mode of action for gibberellin A regulated fiber growth. J Integr Plant Biol. 2016;58(6):577-89. https://doi.org/10.1111/jipb.12429.

46. Huang G, Wu Z, Percy RG, Bai M, Li Y, Frelichowski JE, Hu J, Wang K, Yu JZ, Zhu Y. Genome sequence of Gossypium herbaceum and genome updates of Gossypium arboreum and Gossypium hirsutum provide insights into cotton Agenome evolution. Nat Genet. 2020;52(5):516-24. https://doi.org/10.1038/s41588-020-0607-4.

47. Yoo MJ, Wendel JF. Comparative evolutionary and developmental dynamics of the cotton (Gossypium hirsutum) fiber transcriptome. PLoS Genet. 2014;10(1):e1004073. https://doi.org/10.1371/journal.pgen.1004073.

48. Yuan D, Tang Z, Wang M, Gao W, Tu L, Jin X, Chen L, He Y, Zhang L, Zhu L, et al. The genome sequence of Sea-Island cotton (Gossypium barbadense) provides insights into the allopolyploidization and development of superior spinnable fibres. Sci Rep. 2015:5:17662.

49. Bao Y, Hu G, Flagel LE, Salmon A, Bezanilla M, Paterson AH, Wang Z, Wendel JF. Parallel up-regulation of the profilin gene family following independent domestication of diploid and allopolyploid cotton (Gossypium). Proc Natl Acad Sci U S A. 2011;108(52):21152-7. https://doi.org/10.1073/pnas.1115926109.

50. Tao Y, Zhao X, Mace E, Henry R, Jordan D. Exploring and exploiting pan-genomics for crop improvement. Mol Plant. 2019;12(2):156-69. https://doi.org/10.1016/j.molp.2018.12.016.

51. Danilevicz MF, Tay Fernandez CG, Marsh JI, Bayer PE, Edwards D. Plant pangenomics: approaches, applications and advancements. Curr Opin Plant Biol. 2020;54:18-25. https://doi.org/10.1016/j.pbi.2019.12.005.

52. Bayer PE, Golicz AA, Scheben A, Batley J, Edwards D. Plant pan-genomes are the new reference. Nat Plants. 2020;6(8): 914-20. https://doi.org/10.1038/s41477-020-0733-0.

53. Bolger AM, Lohse M, Usadel B. Trimmomatic: a flexible trimmer for Illumina sequence data. Bioinformatics. 2014;30(15): 2114-20. https://doi.org/10.1093/bioinformatics/btu170.

54. Li H, Durbin R. Fast and accurate short read alignment with Burrows-Wheeler transform. Bioinformatics. 2009;25(14): 1754-60. https://doi.org/10.1093/bioinformatics/btp324.

55. McKenna A, Hanna M, Banks E, Sivachenko A, Cibulskis K, Kernytsky A, Garimella K, Altshuler D, Gabriel S, Daly M, DePristo MA. The genome analysis toolkit: a MapReduce framework for analyzing next-generation DNA sequencing data. Genome Res. 2010;20(9):1297-303. https://doi.org/10.1101/gr.107524.110.

56. Li H, Handsaker B, Wysoker A, Fennell T, Ruan J. The sequence alignment-map format and SAMtools. Bioinformatics. 2009;25(16):2078-9. https://doi.org/10.1093/bioinformatics/btp352.

57. Browning BL, Zhou Y, Browning SR. A one-penny imputed genome from next-generation reference panels. Am J Hum Genet. 2018;103(3):338-48. https://doi.org/10.1016/j.ajhg.2018.07.015.

58. Wang K, Li M, Hakonarson H. ANNOVAR functional annotation of genetic variants from high-throughput sequencing data. Nucleic Acids Res. 2010;38:e164.

59. Falush D, Stephens M, Pritchard JK. Inference of population structure using multilocus genotype data: linked loci and correlated allele frequencies. Genetics. 2003;164(4):1567-87.

60. Jakobsson M, Rosenberg NA. CLUMPP: a cluster matching and permutation program for dealing with label switching and multimodality in analysis of population structure. Bioinformatics. 2007;23(14):1801-6. https://doi.org/10.1093/ bioinformatics/btm233.

61. Retief JD. Phylogenetic analysis using PHYLIP. Methods Mol Biol. 2000;132:243-58. https://doi.org/10.1385/1-59259-1 92-2:243.

62. Yang J, Lee SH, Goddard ME, Visscher PM. GCTA: a tool for genome-wide complex trait analysis. Am J Hum Genet. 2011; 88(1):76-82. https://doi.org/10.1016/j.ajhg.2010.11.011.

63. Danecek P, Auton A, Abecasis G, Albers CA, Banks E, DePristo MA, Handsaker RE, Lunter G, Marth GT, Sherry ST, et al. The variant call format and VCFtools. Bioinformatics. 2011;27(15):2156-8. https://doi.org/10.1093/bioinformatics/btr330.

64. Purcell S, Neale B, Todd-Brown K, Thomas L, Ferreira MAR, Bender D, Maller J, Sklar P, de Bakker PIW, Daly MJ, Sham PC. PLINK: a tool set for whole-genome association and population-based linkage analyses. Am J Hum Genet. 2007;81(3): 559-75. https://doi.org/10.1086/519795.

65. Chen H, Patterson N, Reich D. Population differentiation as a test for selective sweeps. Genome Res. 2010;20(3):393-402. https://doi.org/10.1101/gr.100545.109.

66. Wang Y, Tang H, Debarry JD, Tan X, Li J, Wang X, Lee TH, Jin H, Marler B, Guo H, et al. MCScanX: a toolkit for detection and evolutionary analysis of gene synteny and collinearity. Nucleic Acids Res. 2012;40(7):e49. https://doi.org/10.1093/nar/ gkr11293.

67. Rausch T, Zichner T, Schlattl A, Stutz AM, Benes V, Korbel JO. DELLY: structural variant discovery by integrated pairedend and split-read analysis. Bioinformatics. 2012;28(18):i333-9. https://doi.org/10.1093/bioinformatics/bts378.

68. Chen K, Wallis JW, McLellan MD, Larson DE, Kalicki JM, Pohl CS, McGrath SD, Wendl MC, Zhang QY, Locke DP, et al. BreakDancer: an algorithm for high-resolution mapping of genomic structural variation. Nat Methods. 2009;6(9):677-81. https://doi.org/10.1038/nmeth.1363.

69. Wang X, Zheng Z, Cai Y, Chen T, Li C, Fu W, Jiang Y. CNVcaller: highly efficient and widely applicable software for detecting copy number variations in large populations. Gigascience. 2017;6(12):1-12. https://doi.org/10.1093/giga science/gix115.

70. Poland JA, Bradbury PJ, Buckler ES, Nelson RJ. Genome-wide nested association mapping of quantitative resistance to northern leaf blight in maize. Proc Natl Acad Sci U S A. 2011;108(17):6893-8. https://doi.org/10.1073/pnas.1010894108.

71. Bradbury PJ, Zhang Z, Kroon DE, Casstevens TM, Ramdoss Y, Buckler ES. TASSEL: software for association mapping of complex traits in diverse samples. Bioinformatics. 2007;23(19):2633-5. https://doi.org/10.1093/bioinformatics/btm308.

72. Lippert C, Xiang J, Horta D, Widmer C, Kadie C, Heckerman D, Listgarten J. Greater power and computational efficiency for kernel-based association testing of sets of genetic variants. Bioinformatics. 2014;30(22):3206-14. https://doi.org/10.1 093/bioinformatics/btu504. 
73. Zimin AV, Marçais G, Puiu D, Roberts M, Salzberg SL, Yorke JA. The MaSuRCA genome assembler. Bioinformatics. 2013; 29(21):2669-77. https://doi.org/10.1093/bioinformatics/btt476.

74. Delcher AL, Phillippy A, Carlton J, Salzberg SL. Fast algorithms for large-scale genome alignment and comparison. Nucleic Acids Res. 2002;30(11):2478-83. https://doi.org/10.1093/nar/30.11.2478.

75. Fu L, Niu B, Zhu Z, Wu S, Li W. CD-HIT: accelerated for clustering the next-generation sequencing data. Bioinformatics. 2012;28(23):3150-2. https://doi.org/10.1093/bioinformatics/bts565.

76. Wood DE, Salzberg SL. Kraken: ultrafast metagenomic sequence classification using exact alignments. Genome Biol. 2014;15(3):R46. https://doi.org/10.1186/gb-2014-15-3-r46.

77. Xiao CL, Chen Y, Xie SQ, Chen KN, Wang Y, Han Y, Luo F, Xie Z. MECAT: fast mapping, error correction, and de novo assembly for single-molecule sequencing reads. Nat Methods. 2017;14(11):1072-4. https://doi.org/10.1038/nmeth.4432

78. Walker BJ, Abeel T, Shea T, Priest M, Abouelliel A, Sakthikumar S, Cuomo CA, Zeng Q, Wortman J, Young SK, Earl AM. Pilon: an integrated tool for comprehensive microbial variant detection and genome assembly improvement. PLoS One. 2014;9(11):e112963. https://doi.org/10.1371/journal.pone.0112963.

79. Simao FA, Waterhouse RM, loannidis P, Kriventseva EV, Zdobnov EM. BUSCO: assessing genome assembly and annotation completeness with single-copy orthologs. Bioinformatics. 2015;31(19):3210-2. https:/doi.org/10.1093/bioinformatics/btv351.

80. Chen N. Using RepeatMasker to identify repetitive elements in genomic sequences. Curr Protoc Bioinformatics. 2004; Chapter 4:Unit 4.10.

81. Holt C, Yandell M. MAKER2: an annotation pipeline and genomedatabase management tool for secondgeneration genome projects. BMC Bioinformatics. 2011;12(1):491. https://doi.org/10.1186/1471-2105-12-491.

82. Stanke M, Morgenstern B. AUGUSTUS: a web server for gene prediction in eukaryotes that allows user-defned constraints. Nucleic Acids Res. 2005:33(Web Server):W465-7. https://doi.org/10.1093/nar/gki458.

83. Korf I. Gene finding in novel genomes. BMC Bioinformatics. 2004;5(1):59. https://doi.org/10.1186/1471-2105-5-59.

84. Jones P, Binns D, Chang HY, Fraser M, Li W, McAnulla C, McWilliam H, Maslen J, Mitchell A, Nuka G, Pesseat S, Quinn AF, Sangrador-Vegas A, Scheremetjew M, Yong SY, Lopez R, Hunter S. InterProScan 5: genome-scale protein function classification. Bioinformatics. 2014;30(9):1236-40. https://doi.org/10.1093/bioinformatics/btu031.

85. Golicz AA, Martinez PA, Zander M, Patel DA, Van De Wouw AP, Visendi P, Fitzgerald TL, Edwards D, Batley J. Gene loss in the fungal canola pathogen Leptosphaeria maculans. Funct Integr Genomics. 2015;15(2):189-96. https://doi.org/10.1007/ s10142-014-0412-1.

86. Zhang Z, Li J, Zhao X-Q, Wang J, Wong GK-S, Yu J. KaKs_Calculator: calculating Ka and Ks through model selection and model averaging. Genomics Proteomics Bioinformatics. 2006;4(4):259-63. https://doi.org/10.1016/S1672-0229(07)60007-2.

87. Katoh K, Standley DM. MAFFT multiple sequence alignment software version 7: improvements in performance and usability. Mol Biol Evol. 2013;30(4):772-80. https://doi.org/10.1093/molbev/mst010.

88. Nguyen LT, Schmidt HA, von Haeseler A, Minh BQ. IQ-TREE: a fast and effective stochastic algorithm for estimating maximum-likelihood phylogenies. Mol Biol Evol. 2015;32(1):268-74. https://doi.org/10.1093/molbev/msu300.

89. Li J, Manghwar H, Sun L, Wang P, Wang G, Sheng H, Zhang J, Liu H, Qin L, Rui H, Li B, Lindsey K, Daniell H, Jin S, Zhang $X$. Whole genome sequencing reveals rare off-target mutations and considerable inherent genetic or/and somaclonal variations in CRISPR/Cas9-edited cotton plants. Plant Biotechnol J. 2019;17(5):858-68. https://doi.org/10.1111/pbi.13020.

90. Li J, Wang M, Li Y, Zhang Q, Lindsey K, Daniell H, Jin S, Zhang X. Multi-omics analyses reveal epigenomics basis for cotton somatic embryogenesis through successive regeneration acclimation process. Plant Biotechnol J. 2019;17(2):43550. https://doi.org/10.1111/pbi.12988.

91. Liu Q, Wang C, Jiao X, Zhang H, Song L, Li Y, Gao C, Wang K. Hi-TOM: a platform for high-throughput tracking of mutations induced by CRISPR/Cas systems. Sci China Life Sci. 2019;62(1):1-7. https://doi.org/10.1007/s11427-018-9402-9.

92. Liu H, Li X, Xiao J, Wang S. A convenient method for simultaneous quantification of multiple phytohormones and metabolites: application in study of rice-bacterium interaction. Plant Methods. 2012;8(1):2. https:/doi.org/10.1186/1746-4811-8-2.

93. Li J, Yuan D, Wang P, Wang Q, Sun M, Liu Z, Si H, Xu Z, Ma Y, Zhang B, Pei L, Tu L, Zhu L, Chen LL, Lindsey K, Zhang X, Jin S, Wang M. Cotton pan-genome retrieves the lost sequences and genes during domestication and selection. Datasets. NCBI Bioproject. 2021. https://www.ncbi.nlm.nih.gov/bioproject/PRJNA576032. Accessed 1 Feb 2021.

94. Li J, Yuan D, Wang P, Wang Q, Sun M, Liu Z, Si H, Xu Z, Ma Y, Zhang B, Pei L, Tu L, Zhu L, Chen LL, Lindsey K, Zhang X, Jin $S$, Wang M. Cotton pan-genome retrieves the lost sequences and genes during domestication and selection. Figshare. 2021; https://figshare.com/s/cb3c104782a1dcd90ab0

\section{Publisher's Note}

Springer Nature remains neutral with regard to jurisdictional claims in published maps and institutional affiliations.

\section{Ready to submit your research? Choose BMC and benefit from:}

- fast, convenient online submission

- thorough peer review by experienced researchers in your field

- rapid publication on acceptance

- support for research data, including large and complex data types

- gold Open Access which fosters wider collaboration and increased citations

- maximum visibility for your research: over $100 \mathrm{M}$ website views per year

At BMC, research is always in progress.

Learn more biomedcentral.com/submissions 